\title{
A DISCUSSION ON A RATE-DEPENDENT COHESIVE LAW FOR POLYMER ADHESION INSPIRED FROM EXISTING CONSTITUTIVE EQUATIONS
}

\author{
M. SOLAR \\ ${ }^{1}$ Mechanics of Interfaces and Multiphase Systems (MIM), \\ Institut Charles Sadron (UPR-22), 23 Rue du Loess, 67200 Strasbourg and, \\ INSA Strasbourg, 24 Boulevard de la Victoire, 67000 Strasbourg, France. \\ mathieu.solar@insa-strasbourg.fr
}

Key words: polymer, glassy, surface separation, MD simulations, debonding, cohesive law.

\begin{abstract}
The failure of adhesion between two polymers is a complex phenomenon involving bulk dissipation (i.e., shear yielding, cavitation and crazing), surface adhesion (through Van der Waals interactions) and connector chain pull-out. This article is concerned with a cohesive model for polymer surface separation, which incorporates connector pull-out since it is attempted to adjust this model on coarse-grained molecular dynamics (MD) simulations. This model is made of a viscoplastic constitutive equation taking inspiration from the works by Anand and Gurtin (2003). The MD simulations performed and here analysed were done in close collaboration with Prof. Erik van der Giessen (MuMec, Zernike Institute for Advanced Materials, University of Groningen, Groningen, the Netherlands).
\end{abstract}

\section{Introduction}

Fracture toughness and the fracture mechanisms of planar polymer interfaces have been the subject of extensive experimental research, aiming to bridge the gap between fracture mechanics and polymer physics. Examples of still unresolved issues are the problem of craze failure near the tip of a crack embedded inside a craze and the failure of adhesion between immiscible polymers stitched together with block copolymers $[1,2,3,4,5,6,7]$. The failure of adhesion between two polymers is a complex phenomenon involving: (i/a), surface adhesion through Van der Waals interactions (adhesion, adherence and surface tension); (i/b), the pull-out of polymer chains across the interface by means of forced reptation; (ii), bulk dissipation taking place on either side of the interface by shear yielding, cavitation and crazing. Chain scission is another potentially important mechanism, but is not considered here. Furthermore, the latter is the basis of the mechanism of "crack propagation" which is hardly modelized by atomistic approaches. In this situation, the phase field methods are relevant and, may be able to contribute as well [8]. As an example, nice review on polymer adhesion can be found in $[9,10]$ and, some theoretical predictions for interfacial debonding in layered elastic composites exist in the literature, see Ref. [11]. Bulacu and Van der Giessen have reported on coarse-grained MD simulations of the adhesion between two glassy polymers reinforced with connector chains, which focused entirely on the process of connector pull-out $[12,13]$. The results gave promising conclusions about the effects on the adhesive strength of different connector architectures and external debonding conditions (separation rate and temperature), but ignored 
the contribution of the viscoelastic deformation inside the polymers on either side of the interface. This last mechanism can be very important as the strain rate, the areal density or the connector chain length increase, and is not so obvious to determinate. This paper describes a potentially realistic cohesive law for interface separation, where the mechanical behavior during interface opening is extracted from MD results for pull-out process between polymer interfaces. This law is aimed to be used for crack growth calculations using Finite Element simulations. This contribution completes the previous work of Solar and Van der Giessen [14] and Solar [15], where a first insight of the competition between surface and bulk phenomena, involved in polymer interface separation problems with low areal density of connecting molecules (i.e., the so-called mushroom regime), was presented for various separation velocities.

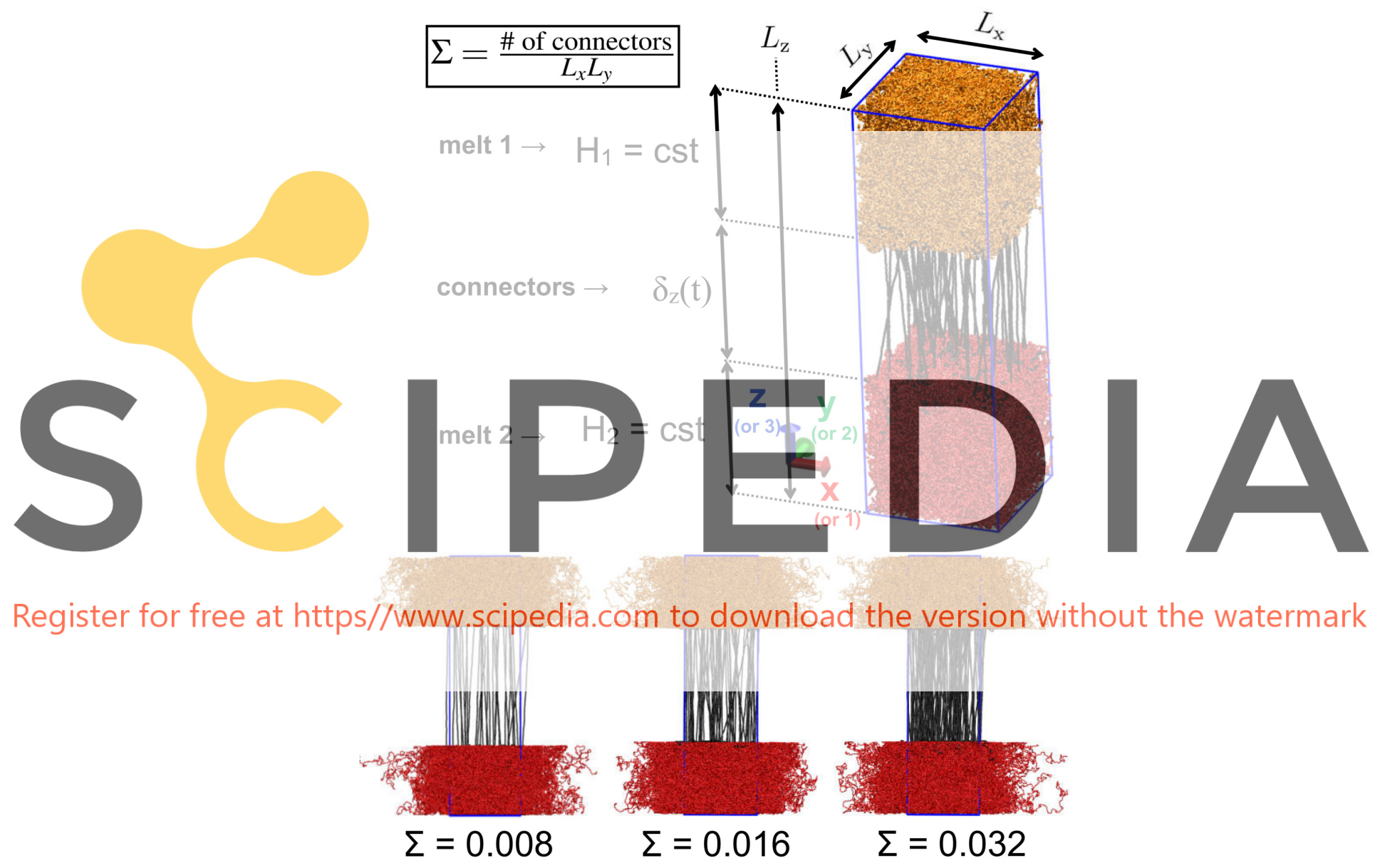

Figure 1: (Color online) Straining of a coarse-grained system with two melts (consisting of 385 chains, each having 500 beads) linked together by $N_{\mathrm{c}}^{\text {conn }}=30 ; 60 ; 120$ connectors respectively, each of $N_{\mathrm{b}}^{\text {conn }}=150$ beads, see Refs. $[14,15]$. The system is pulled at both sides with a velocity $v_{\text {pull }} / 2$. Vertical traction $T_{n}$ is an overall measure of the vertical traction in the MD box. The three snapshots depicts the system after stretching at $\dot{\varepsilon}_{\text {eng }}=0.005$, when $T_{n}$ reaches $\sigma_{\mathrm{coh}}$. 


\section{MD simulations of polymer adhesion}

Two amorphous polymer melts, stitched together by distinct connector molecules, are studied. This system is contained in an (initially orthorhombic) MD cell with a given cell matrix $H^{\text {cell }}$, see Fig. 1 and Ref. [16]. The system is deformed by imposing the straining of the MD cell through a uniform rate of deformation gradient. The principles of the analysis were already introduced in Refs. $[14,15]$. One reminds that the type of simulations being performed were (a) full debonding (b), pure pull-out and (c), bulk flow. We focused on the pure pull-out process only. The macroscopic kinematics was implemented to have a deformation such as $\Delta L_{z}:=L_{z}-L_{z, 0}=\mathrm{v}_{\text {pull }} t$, where $\Delta L_{z}$ is termed as the separation between the two walls at $z=+L_{z} / 2$ and $z=-L_{z} / 2{ }^{1}$. The interface kinematics was analysed using a so-called cohesive opening, denoted by $\Delta_{\mathrm{n}}$. This quantity looks like a nano-crack opening, but the previous naming was preferred. The cohesive opening was estimated by the mean of vertical thickness estimations, computed for the two melts, the connectors and at every time of the simulation. Such timeresolved diagrams require the calculation of density profiles, see Refs. [14, 15]. Once this is performed, the cohesive opening $\Delta_{\mathrm{n}}$ and the opening rate $\dot{\Delta}_{\mathrm{n}}$ are calculated according to the following relations

$$
\begin{aligned}
& \Delta_{\mathrm{n}}=\delta_{z}-\delta_{z, 0}=\left(L_{z}-L_{z, 0}\right)-2\left(H_{z}-H_{z, 0}\right), \\
& \dot{\Delta}_{\mathrm{n}}=\dot{\delta}_{z}=\dot{L}_{z}-2 \dot{H}_{z},
\end{aligned}
$$

where $H_{z}=H_{z, 0}=$ cst. (resp. $H_{z, 0}$ ) stands for the averaged thickness of the two melts (resp. their initial vertical thickness) and, where $\dot{H}_{z}=0$ since one considers pure pull-out MD simulations. As an

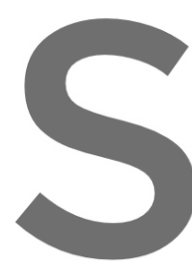
indication to the reader, of the energy $W$ dissipa $L_{z, 0}$ and $\dot{\varepsilon}_{\text {eng }}$ are consta opening $\Delta_{\mathrm{n}}$, see Fig. 2. dependencies of $T_{\mathrm{n}}$ (resp.
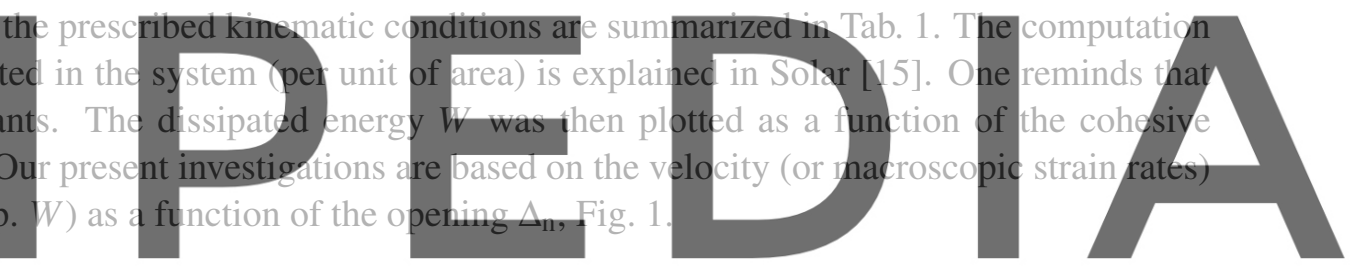

Register for free at https//www.scipedial.commto downlomokthe version without the watermark

\begin{tabular}{|l|l|l|l|}
0.002 & 0.254 & $=v_{\text {pull }}$ & 0.056 \\
0.003 & 0.381 & $=v_{\text {pull }}$ & 0.085 \\
0.004 & 0.508 & $=v_{\text {pull }}$ & 0.113 \\
0.005 & 0.635 & $=v_{\text {pull }}$ & 0.141 \\
0.010 & 1.270 & $=v_{\text {pull }}$ & 0.282 \\
\hline
\end{tabular}

Table 1: Prescribed kinematic conditions, where one knows that $v_{\text {pull }}=\dot{\varepsilon}_{\text {eng }} L_{z, 0}$ and that $\dot{\Delta}_{\mathrm{n}} / \delta_{\text {gap }}=28 \dot{\varepsilon}_{\text {eng }}$.

\footnotetext{
${ }^{1}$ The (engineering) vertical strain rate $\dot{\varepsilon}_{\text {eng }}=\dot{\varepsilon}_{33}$ defines the vertical pulling velocity as being $v_{\text {pull }}=\dot{\varepsilon}_{\text {eng }} L_{z, 0}$ for pull-out simulations (the melts remain none-deformed), whereas it defines the effective vertical pulling velocity $v_{\text {pull }}$ for total debonding simulations (the melts are deformed).
} 


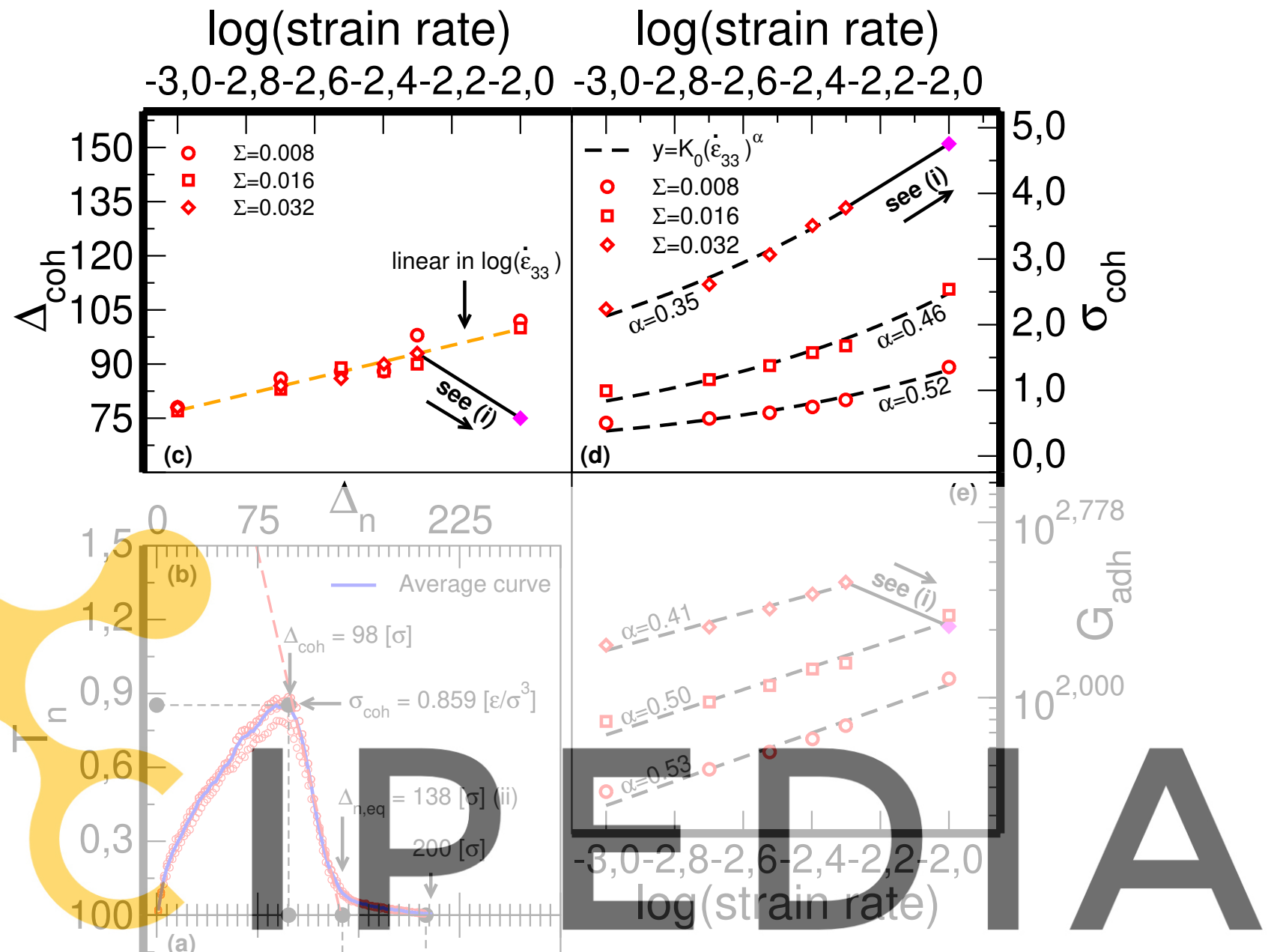

(i) chain scission

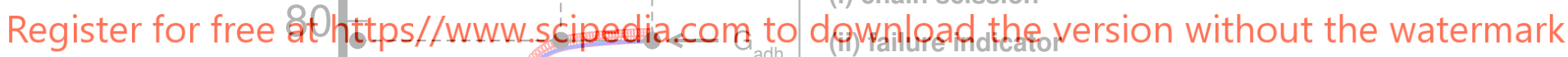

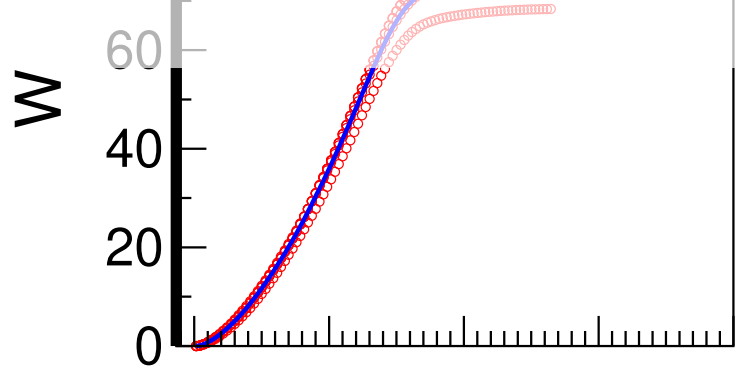

Figure 2: (Color online) Pull-out MD simulation results summarized as a function of $\log \left(\dot{\varepsilon}_{\text {eng }}\right)$ : $\operatorname{traction} T_{n}$ (resp. energy $W$ ) versus separation response $\Delta_{\mathrm{n}}$ obtained from five MD runs (symbols) in panel (a) (resp. panel (b)). The smooth curve is the average response, with corresponding characteristics of peak and failure indicated. The extracted quantities (i.e., $\Delta_{\text {coh }}, \sigma_{\text {coh }}$ and $G_{\text {adh }}$ ) are given in panels (c), (d) and (e) for all the strain rates and the areal densities investigated, see Refs. [14, 15]. 


\section{A short discussion on the Universal Binding Law}

The Universal Binding Law, originally proposed by Rose et al. [37], has proved to be a powerful approach to describe the normal response of many metallic and covalently bonded materials. In this approach, the interface energy expression, i.e.

$$
W\left(\Delta_{\mathrm{n}}\right)=G_{\text {adh }}\left\{1-\left[\left(1+\frac{\Delta_{\mathrm{n}}}{\Delta_{\text {coh }}}\right) \exp \left(-\frac{\Delta_{\mathrm{n}}}{\Delta_{\text {coh }}}\right)\right]\right\},
$$

serves as a potential, from which the normal traction is obtained through $T_{\mathrm{n}}=\partial W / \partial \Delta_{\mathrm{n}}$, that is

$$
T_{\mathrm{n}}\left(\Delta_{\mathrm{n}}\right)=\frac{G_{\mathrm{adh}}}{\Delta_{\mathrm{coh}}}\left(\frac{\Delta_{\mathrm{n}}}{\Delta_{\mathrm{coh}}}\right)\left(\exp \left[-\frac{\Delta_{\mathrm{n}}}{\Delta_{\mathrm{coh}}}\right]\right),
$$

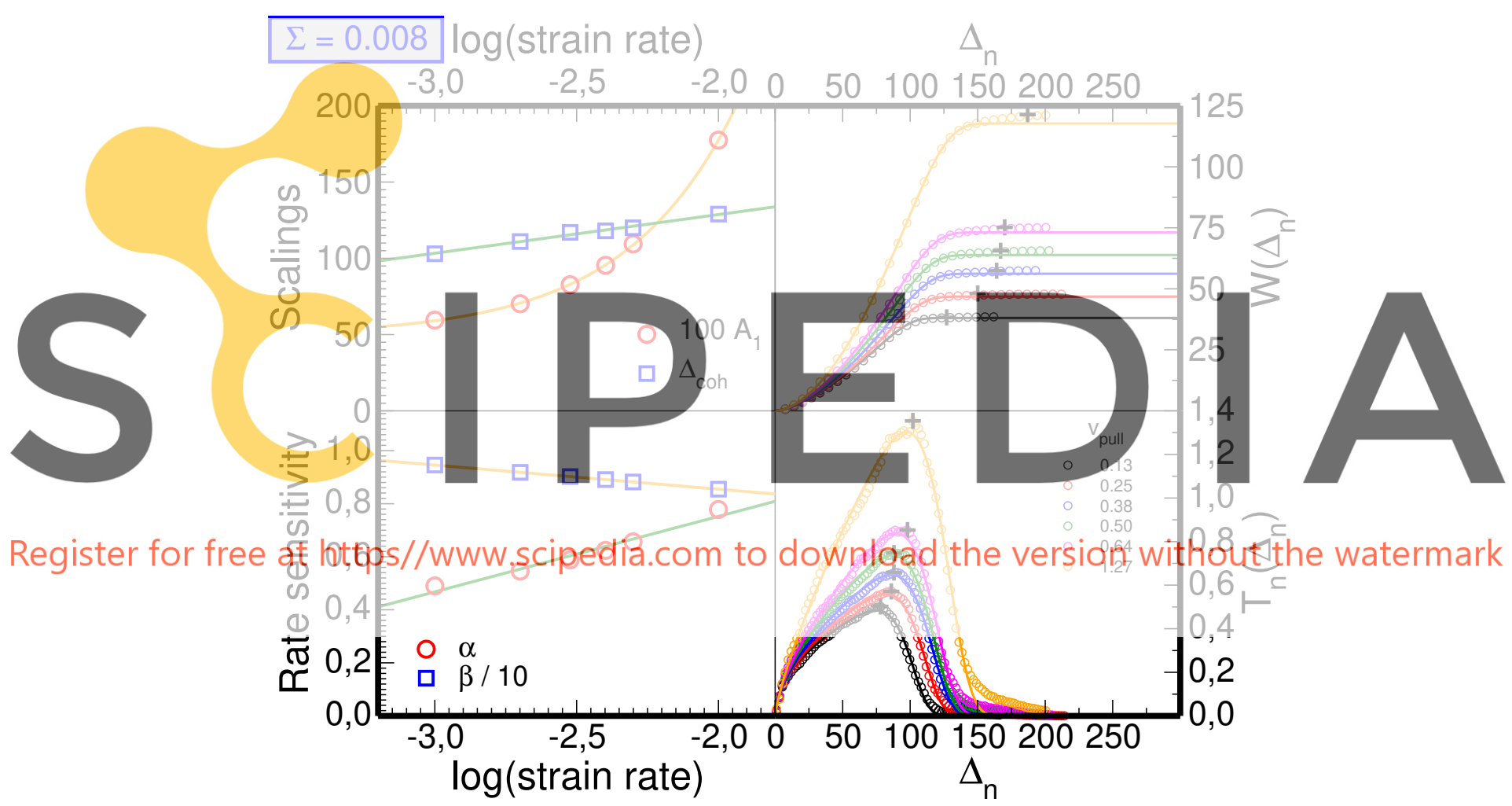

Figure 3: (Color online) Attempt to fit the MD interface response (in pull-out) by the function at Eq. (5). The agreement between MD data (symbols) and model (lines) is excellent, but the sensitivity exponents deviate strongly from the Universal Binding Law $(\alpha=\beta=1)$. The areal density is $\Sigma=0.008 \sigma^{-2}$.

where $\Delta_{\text {coh }}$ is a characteristic length scale of the interface separation behavior. In Eq. (3), $G_{\text {adh }}$ is the total adhesion energy, i.e. the area under the $T_{\mathrm{n}}$ vs $\Delta_{\mathrm{n}}$ curve. In addition to this original formulation, we can consider the following simple extension which consists in a bell-shaped function, used to capture the stress - separation length behavior of the interface, rather than postulating the existence of a welldefined potential $W$. In this extension, the position of the peak and the debonding length can be adjusted 
both separately. This basic picture leads us to introduce the following function, which consists in a generalization of the Universal Binding Energy Relation (UBER)

$$
T_{\mathrm{n}}\left(\Delta_{\mathrm{n}}\right)=A_{1}\left(\frac{\Delta_{\mathrm{n}}}{\Delta_{\mathrm{coh}}}\right)^{\alpha}\left(\exp \left[-\left(\frac{\Delta_{\mathrm{n}}}{\Delta_{\mathrm{coh}}}\right)^{\beta}\right]\right),
$$

where $A_{1}, \Delta_{\text {coh }}, \alpha$ and $\beta$ are material parameters used to fix the shape of the curve. The cohesive strength $\sigma_{\text {coh }}$ would still be defined as the maximum of the " $T_{\mathrm{n}} v s . \Delta_{\mathrm{n}}$ " curve. The energy $W$ - separation length $\Delta_{\mathrm{n}}$ behavior would be determined then by integration of Eq. (5). In Fig. 3, one proposes an attempt to fit the MD interface response at low areal density (in pull-out) by the extended Universal Binding law at Eq. (5). Nevertheless, this kind of cohesive description of the interface separation process was disregarded in this contribution, since one obtains as many set of fitting parameters $\left(A_{1} ; \Delta_{\text {coh }} ; \alpha ; \beta\right)$ as there are curves to be fit, specially for the strain-rate dependence. In addition, the strain rate sensitivity is hidden is the fitting-parameters, see Fig. 3.

\section{A Rate-Dependent formulation of the cohesive law}

One address here a rate-dependent plastic cohesive law for polymer surface separation, motivated by the plasticity theory for amorphous polymers proposed by Anand and Gurtin [41], based on a complex kinematic hardening description of viscoplasticity. The latter is aimed to describe plasticity in polymers, which could involve more complex molecular mechanisms as the polymer molecule extraction during

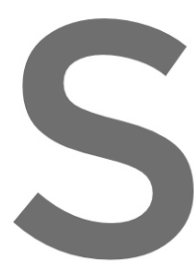
surface separation does.
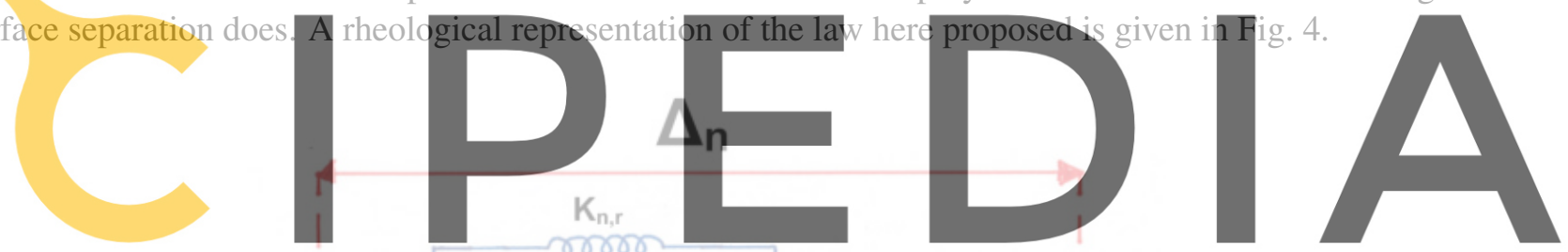

Register for free at https//www.scipedia.com to, download the version without the watermark<smiles></smiles><smiles>C1CCCCC1</smiles>
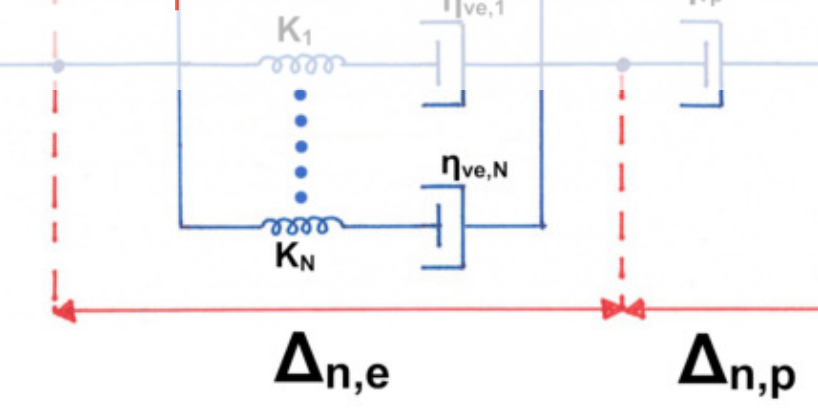

Figure 4: (Color online) Schematic representation of the here-presented viscoelasto-viscoplastic cohesive law for polymer adhesion with $N$ viscoelastic branches. The most simple situation is when the viscoelastic relaxation is characterized by a single relaxation time $\tau_{\mathrm{r}}$ and, is materialized through a single linear dashpot of viscosity $\eta_{\mathrm{ve}}$. One can find the following relation: $\tau_{\mathrm{r}}=\eta_{\mathrm{ve}} /\left(K_{\mathrm{n}, \mathrm{u}}-K_{\mathrm{n}, \mathrm{r}}\right)$. As the interface opens, the viscoplasticity sets in during the creep of a none-linear dashpot of viscosity $\eta_{\mathrm{vp}}$, see Eq. (11). The behavior of this last one originates from the model by Anand and Gurtin. 


\subsection{Constitutive formulation and governing equations}

An additive decomposition of the opening displacement rate $\dot{\Delta}_{\mathrm{n}}$ into a viscoelastic $\dot{\Delta}_{\mathrm{n}, \mathrm{e}}$ and a viscoplastic $\dot{\Delta}_{\mathrm{n}, \mathrm{p}}$ part is chosen as a standard vision of viscoplasticity. It follows then that

$$
\dot{\Delta}_{\mathrm{n}}=\dot{\Delta}_{\mathrm{n}, \mathrm{e}}+\dot{\Delta}_{\mathrm{n}, \mathrm{p}},
$$

where the constitutive equations must also be supplied. For tensile verticale opening of an interface, the vertical traction $T_{\mathrm{n}}$ is positive.

\subsubsection{Recoverable elastic behavior of the interface}

The recoverable elastic behavior of the interface may be described in two different fashions. One replaces the other and vice versa, since the first fashion concerns instantaneous elasticity and the second fashion deals with elasticity using a relaxation time spectrum. These both description cannot be implemented at the same time in principles. The elastic (and rate independent) mechanical behavior of an interface is described by the following standard relation

$$
\dot{\Delta}_{\mathrm{n}, \mathrm{e}}=\dot{T}_{\mathrm{n}} / K_{\mathrm{n}},
$$

in terms of an (instantaneous) interface stiffness $K_{\mathrm{n}}$. This description of elasticity is simple, relatively stable during numerical integration of the constitutive equation but, does not take into account the dopendence of the elastic re of the opening rate. To

Opening rate dependent elasticity A viscoelast
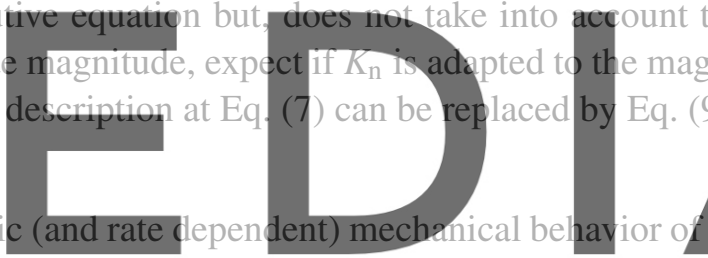

terface can be described by a multi-relaxation time description, where the viscoelasticity of the interface

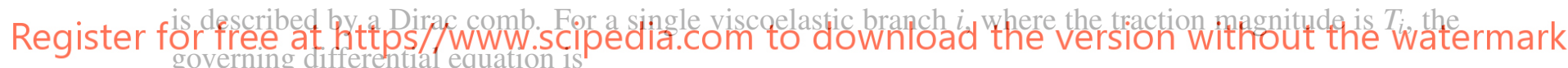
governing differential equation is

$$
\dot{\Delta}_{\mathrm{n}, \mathrm{e}}=\left(\frac{1}{K_{i}}\right) \cdot \dot{T}_{i}+\left(\frac{1}{K_{i} \tau_{\mathrm{r}, i}}\right) \cdot T_{i}
$$

where $K_{i}=K_{\mathrm{n}, \mathrm{u}, i}-K_{\mathrm{n}, \mathrm{r}}$ is the stiffness of the branch $i$ and, the relaxation time of the branch is given by $\tau_{\mathrm{r}, i}=\eta_{\mathrm{ve}, i} / K_{i}$. This differential equation results in the additive response of a spring in serie with a dashpot. If $N$ viscoelastic branches are present, the set of equations to solve is

$$
\left\{\begin{aligned}
\sum_{i=1}^{N} T_{i} & =T_{\mathrm{n}}-K_{\mathrm{n}, \mathrm{r}} \Delta_{\mathrm{n}, \mathrm{e}} \quad \text { and, } \quad \sum_{i=1}^{N} \dot{T}_{i}=\dot{T}_{\mathrm{n}}-K_{\mathrm{n}, \mathrm{r}} \dot{\Delta}_{\mathrm{n}, \mathrm{e}} \\
\dot{\Delta}_{\mathrm{n}, \mathrm{e}} & =\left(\frac{\tau_{\mathrm{eq}}}{\eta_{\mathrm{eq}}^{\tau}}\right) \cdot \dot{T}_{\mathrm{n}}+\left(\frac{1}{\eta_{\mathrm{eq}}^{\tau}}\right) \cdot T_{\mathrm{n}}-\left(\frac{K_{\mathrm{n}, \mathrm{r}}}{\eta_{\mathrm{eq}}^{\tau}}\right) \cdot \Delta_{\mathrm{n}, \mathrm{e}}-\sum_{i=1}^{N} \sum_{j \neq i}\left(\frac{\tau_{\mathrm{r}, i}}{\eta_{\mathrm{eq}}^{\tau}}\right) \cdot \dot{T}_{j} \\
\eta_{\mathrm{eq}}^{\tau} & =\sum_{i=1}^{N}\left[\left(K_{i}+K_{\mathrm{n}, \mathrm{r}}\right) \cdot \tau_{\mathrm{r}, i}\right] \quad ; \quad \tau_{\mathrm{eq}}=\sum_{i=1}^{N} \tau_{\mathrm{r}, i}
\end{aligned}\right.
$$


where the third equation comes out from the two first equations and Eq. (8). In case of a single-relaxation time description, the set of equations at (9) can be considerably simplified and reduces to

$$
\dot{\Delta}_{\mathrm{n}, \mathrm{e}}=\frac{1}{K_{\mathrm{n}, \mathrm{u}}}\left(\dot{T}_{\mathrm{n}}+\frac{1}{\tau_{\mathrm{r}}} T_{\mathrm{n}}\right)-\left(\frac{1}{\tau_{\mathrm{r}}} \frac{K_{\mathrm{n}, \mathrm{r}}}{K_{\mathrm{n}, \mathrm{u}}}\right) \Delta_{\mathrm{n}, \mathrm{e}},
$$

in terms of an instantaneous (resp. relaxed) interface stiffness $K_{\mathrm{n}, \mathrm{u}}\left(\right.$ resp. $K_{\mathrm{n}, \mathrm{r}}$ ). The condition $K_{\mathrm{n}, \mathrm{r}}<K_{\mathrm{n}, \mathrm{u}}$ is necessary to stay within the framework of Thermodynamics. The elastic opening-rate sensitivity is regulated through the single relaxation time $\tau_{\mathrm{r}}$, or a relaxation time spectrum, if many branches are connected in parallel. The previous constants are positive-definite. This description of elasticity is more complex, takes simply into account a possible variation of the elastic regime onto the opening rate magnitude but, can be less stable during the numerical integration. One should notice that Eq. (10) contains the pure elastic behavior in its asymptotic limits: (a) if $\tau_{\mathrm{r}}$ tends to $+\infty$, the viscoelastic behavior relaxes infinitely slowly to its relaxed state, as if it was purely elastic of stiffness $K_{\mathrm{n}}=K_{\mathrm{n}, \mathrm{u}}$ in Eq. (7); (b) if $\tau_{\mathrm{r}}$ tends to 0 , the viscoelastic behavior relaxes infinitely fastly to its relaxed state and reduces to " $T_{\mathrm{n}}=K_{\mathrm{n}, \mathrm{r}} \Delta_{\mathrm{n}, \mathrm{e}}$ ", as if it was purely elastic of stiffness $K_{\mathrm{n}}=K_{\mathrm{n}, \mathrm{r}}$ in Eq. (7). In that sense, this viscoelastic-viscoplastic model recovers and improves nicely the elasto-viscoplastic formulation.

\subsubsection{Irreversible viscoplastic behavior of the interface}

The plastic (and rate dependent) mechanical behavior of the interface may be, (a) an additive combination

of classical weighted Eyting

the two forms below
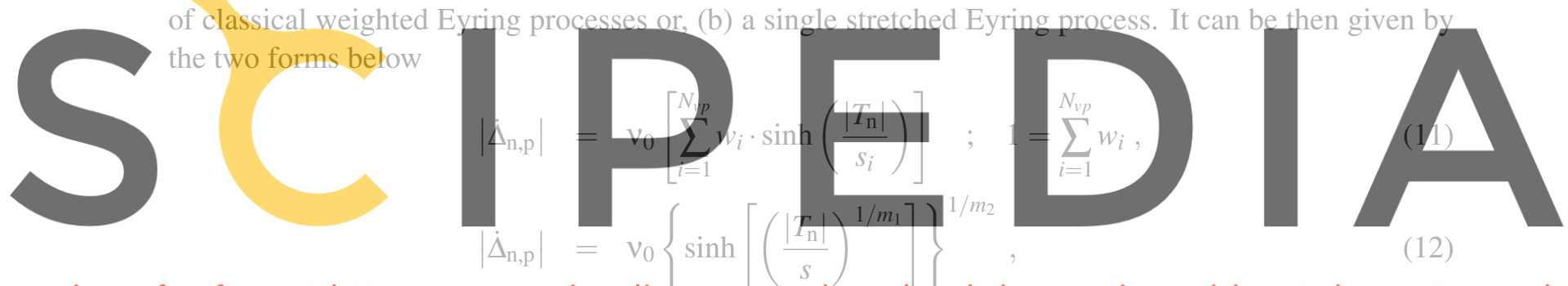

(12)

Register for free at https//www.scipedia.com to downtoad the version without the watermark in terms of a set of weights $w_{i}$, a reference plastic opening rate $v_{0}$, two rate sensitivity coefficients $m_{1}$ and $m_{2}$ and a variable interfacial resistance $s$. Since the form at Eq. (11) possesses potentially a huge range of degrees of freedom, the form at Eq. (12) was preferred first. Taking inspiration from the works by Anand et al. [41], the interface plastic resistance is taken to evolve with the permanent opening according to the following evolution equations (like a relaxation time spectrum for viscoelasticity does),

$$
\begin{aligned}
& \dot{\eta}(t)=g_{0}\left[\frac{s(t)}{s_{\mathrm{cv}}}-1\right]\left|\dot{\Delta}_{\mathrm{n}, \mathrm{p}}\right|, \\
& \dot{s}(t)=h_{0}\left[1-\frac{s(t)}{\tilde{s}(\eta)}\right]\left|\dot{\Delta}_{\mathrm{n}, \mathrm{p}}\right|, \\
& \tilde{s}(\eta)=s_{\mathrm{cv}}\left[1+b\left[\eta_{\mathrm{cv}}-\eta(t)\right]\right] .
\end{aligned}
$$

The parameter list increases then and, one must add: $h_{0}, g_{0}, s_{\mathrm{cv}}, b, s_{0}$ and $\eta_{\mathrm{cv}}$. A last useful detail concerns the initial conditions for the internal variables $s=s(0)$ and $\eta=\eta(0)$. From Anand and Gurtin, these initial conditions are restricted to the following conditions: $s(0)=s_{0}, \eta(0)=0$ and

$$
s_{0} \leq s(t) \leq s_{\mathrm{cv}}\left(1+b \eta_{\mathrm{cv}}\right) .
$$


This last condition was disregarded in our contribution, since it enforces that $s_{\mathrm{cv}}>s_{0}$. One decided to unlock this constraint first, to supplement the model with an additional degree-of-freedom. Thus, the phase portrait might be different from the one described in the work of Anand and Gurtin. The variable $\tilde{s}$ is aimed to play the role of a saturation value of $s$ (if $s>\tilde{s}$ then $\dot{s}<0$, whereas if $s<\tilde{s}$ then $\dot{s}>0$ ).

\subsubsection{Some aspects on the numerical integration}

To simplify the presentation of the equations presented hereafter, let us now introduce the following set of variables

$$
\bar{\kappa}_{1}=\frac{\bar{\kappa}_{3}}{\tau_{\mathrm{r}}} \quad, \quad \bar{\kappa}_{2}=\frac{K_{\mathrm{n}, \mathrm{r}}}{K_{\mathrm{n}, \mathrm{u}}} \quad \text { and }, \quad \bar{\kappa}_{3}=\frac{1}{K_{\mathrm{n}, \mathrm{u}}} \frac{\left|T_{\mathrm{n}}\right|}{\left|\dot{\Delta}_{\mathrm{n}, \mathrm{p}}\right|} .
$$

Using Eqs. (6) and (7) (for elasticity) or Eqs. (6) and (10) (for viscoelasticity), one can further specify the viscoelastic and viscoplastic opening rates at time $t$ as firstly,

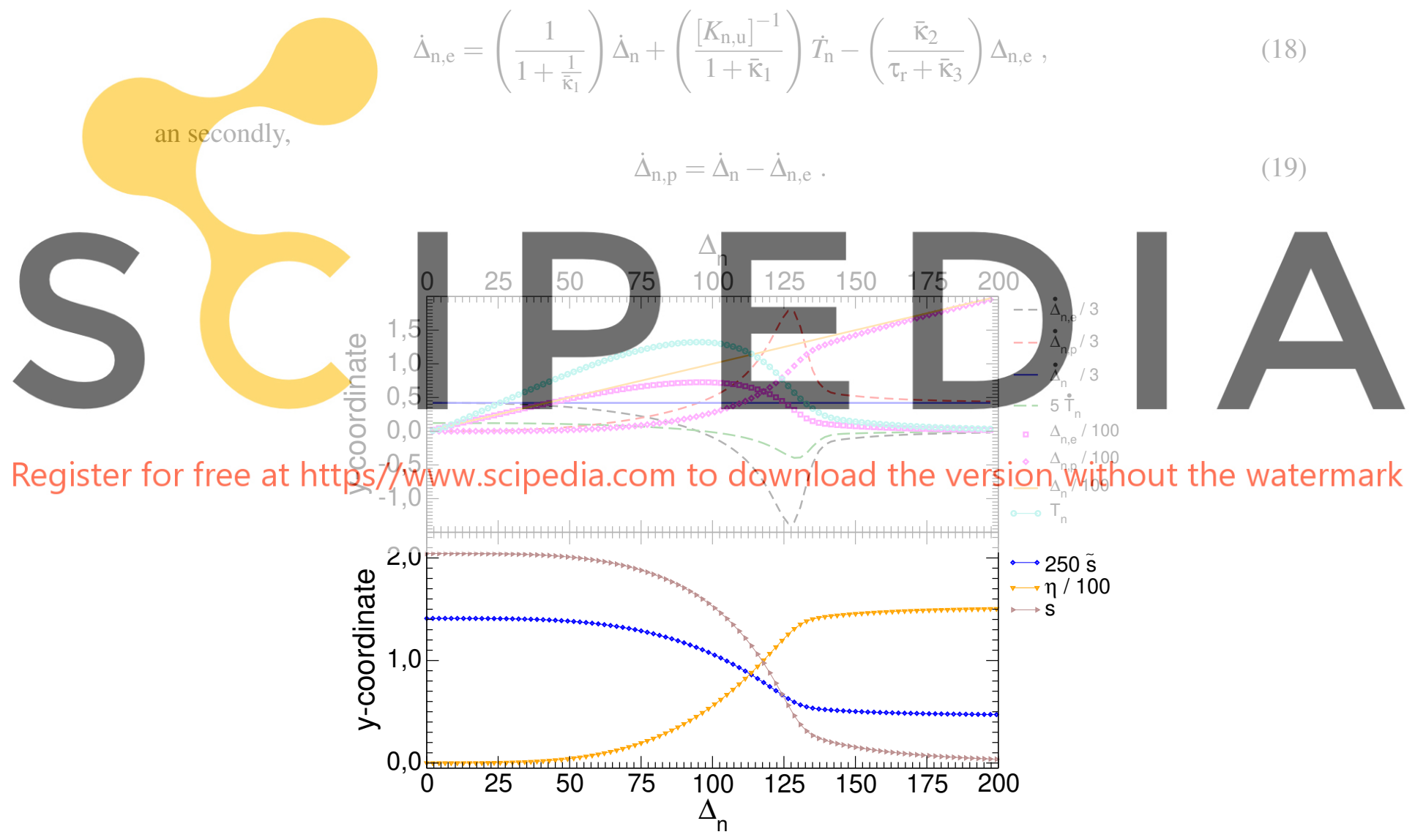

Figure 5: (Color online) Integration of the cohesive law for an engineering macroscopic strain rate $\dot{\varepsilon}_{\text {eng }}$ of 0.010 . The material parameters are given in Tab. 2. The sampling for the curve is 94 points. The evolution of the internal variables are presented in this figure, match initial conditions and seems to have smooth evolutions as the interface opening sets in. Stable integration conditions seems to have been reached, but this issue will be tested more in details in further contributions. 
One clearly remarks that if $\tau_{\mathrm{r}}$ tends to 0 (i.e., a purely elastic interface of stiffness $K_{\mathrm{n}}=K_{\mathrm{n}, \mathrm{r}}$ ), then $\bar{\kappa}_{1}$ tends to $+\infty$. At the same time, the asymptotic behavior of Eq. (18) becomes exactly the one expected for a purely elasto-viscoplastic interface. Thus, Eqs. (18) and (19) are aimed to be universal to describe our elasto-viscoplastic or viscoelasto-viscoplastic interface behaviors. One continues then the description by the updating equation for the traction rate at time $t+\delta t$. For the full visco-elasto-plastic interface, it was chosen as to be

$$
\dot{T}_{\mathrm{n}}(t+\delta t)=\left(K_{\mathrm{n}, \mathrm{u}}\right) \dot{\Delta}_{\mathrm{n}, \mathrm{e}}(t)+\left(\frac{K_{\mathrm{n}, \mathrm{r}}}{\tau_{\mathrm{r}}}\right) \Delta_{\mathrm{n}, \mathrm{e}}(t)-\frac{T_{\mathrm{n}}(t)}{\tau_{\mathrm{r}}},
$$

whereas it was slightly modified into,

$$
\dot{T}_{\mathrm{n}}(t+\delta t)=\left(K_{\mathrm{n}, \mathrm{r}}\right) \dot{\Delta}_{\mathrm{n}, \mathrm{e}}(t)=\left(K_{\mathrm{n}}\right) \dot{\Delta}_{\mathrm{n}, \mathrm{e}}(t),
$$

for the purely elasto-viscoplastic interface, since the term " $1 / \tau_{\mathrm{r}}$ " may cause instabilities during the integration, if the magnitude of $\tau_{\mathrm{r}}$ becomes $\ll 1$. Since the cohesive law presented herein is viscoplastic, the fourth Runge-Kutta method should be suitable to integrate the cohesive law from $t$ to $t+\delta t$ and, the visco-elastic and visco-plastic rates were calculated using Eqs. (18) and (19).
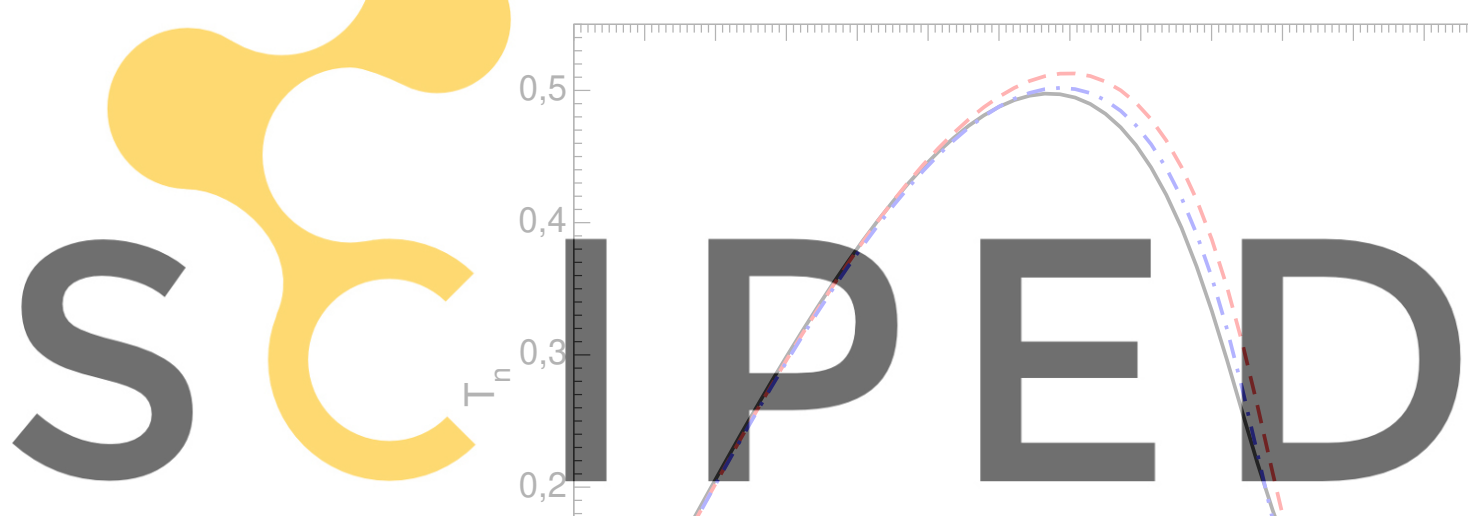

Register for free at https//n
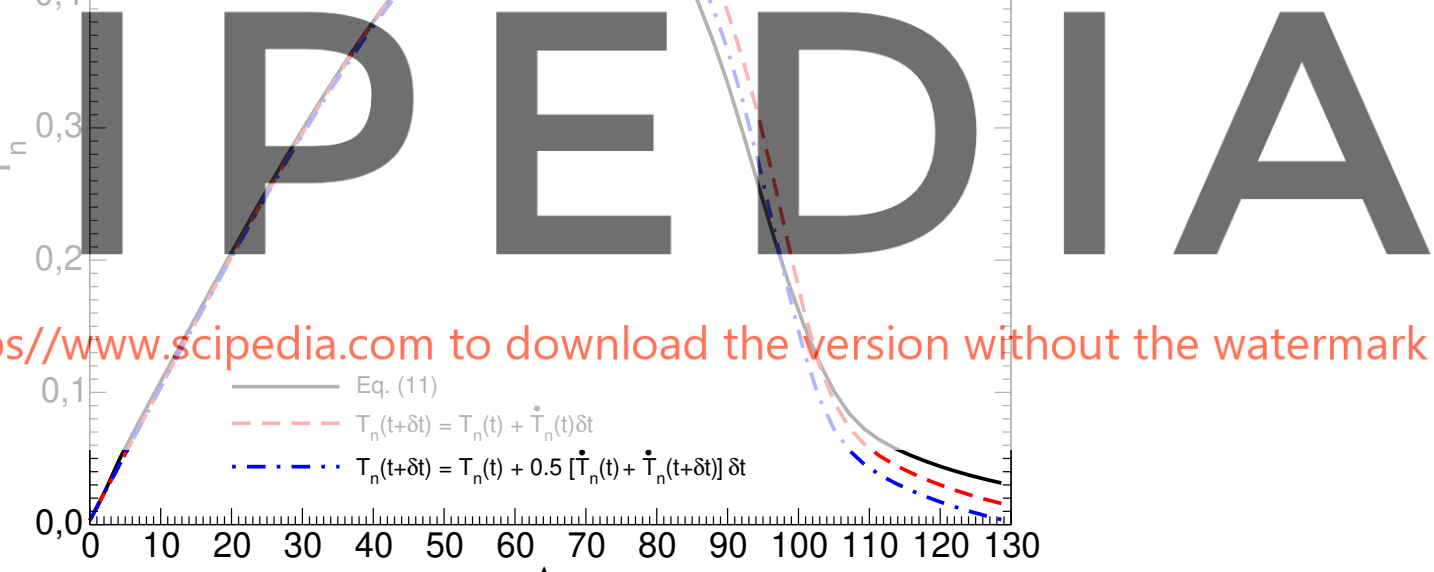

$\cdots-\cdots T_{n}(t+\delta t)=T_{n}(t)+0.5\left[\dot{T}_{n}(t)+\dot{T}_{n}(t+\delta t)\right] \delta t$

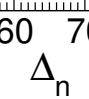

Figure 6: (Color online) Integration of the cohesive law for an engineering strain rate $\dot{\varepsilon}_{\text {eng }}$ of 0.010 (see Fig. 5) for three methods of updating the vertical traction, the time increment $\delta t$ being the same.

The initial conditions being needed to start the integration are provided below, that is

$$
\begin{array}{rll}
T_{\mathrm{n}}(0)=0 & \text { and, } & \dot{\Delta}_{\mathrm{n}, \mathrm{e}}(0)=\dot{\Delta}_{\mathrm{n}}(0) \\
\dot{\Delta}_{\mathrm{n}, \mathrm{p}}(0)=0 & \text { and, } & \dot{T}_{\mathrm{n}}(0)=\left(K_{\mathrm{n}, \mathrm{u}}\right) \dot{\Delta}_{\mathrm{n}, \mathrm{e}}(0)
\end{array}
$$

As the Runge-Kutta method is applied, one updates the internal variables $\eta, \tilde{s}, s$ and $\bar{\kappa}_{3}$ at the same time. As an example in Fig. 5, one plotted this cohesive model for an engineering macroscopic strain 
rate $\dot{\varepsilon}_{\text {eng }}$ of 0.010 , or $\dot{\Delta}_{\mathrm{n}} \approx 1.3$ (equivalent in pull-out), see Tab. 1 . The values of the material parameters are indicated in Tab. 2. One must notices that this example stands for the case of an elasto-viscoplastic interface law, for which one decided to update the vertical traction $T_{\mathrm{n}}(t+\delta t)$ using Eq. (11) instead of an Euler explicit first order scheme. Nevertheless, one turns our attention back in favor of an Euler explicit first order scheme for the case of the full visco-elasto-plastic cohesive law. A velocity-Verletlike first order scheme is also suitable. Fig. 6 illustrates the slight difference, that may happen during integration of the constitutive equation: it is acceptable to our opinion and, our integration procedure appears reproducible. Furthermore, Fig. 6 justifies that parameter values found for the elasto-viscoplastic interface law may be re-used as initial guesses for the full viscoelasto-viscoplastic interface law.

\subsection{Partial dimensional analysis of the model}

Plasticity theories for amorphous polymers, like proposed by Anand and Gurtin [41] and the one presented here, are clever-minded but contain many fitting parameters. None-linear regression reduces then to find a well-optimized set of parameters, where the residue between the model and the data is smallest as possible. Nevertheless, this residue function is a multi-minima function and, it may happens that the parameter optimization is frozen in a minimum, which is not the well-optimized minimum (if this minimum exists). One used a fitting procedure inspired directly from the principle of least action, where fitting parameters evolve like beads in an MD box. More details will be given in an other contribution, but a brief discussion is proposed in App. A.

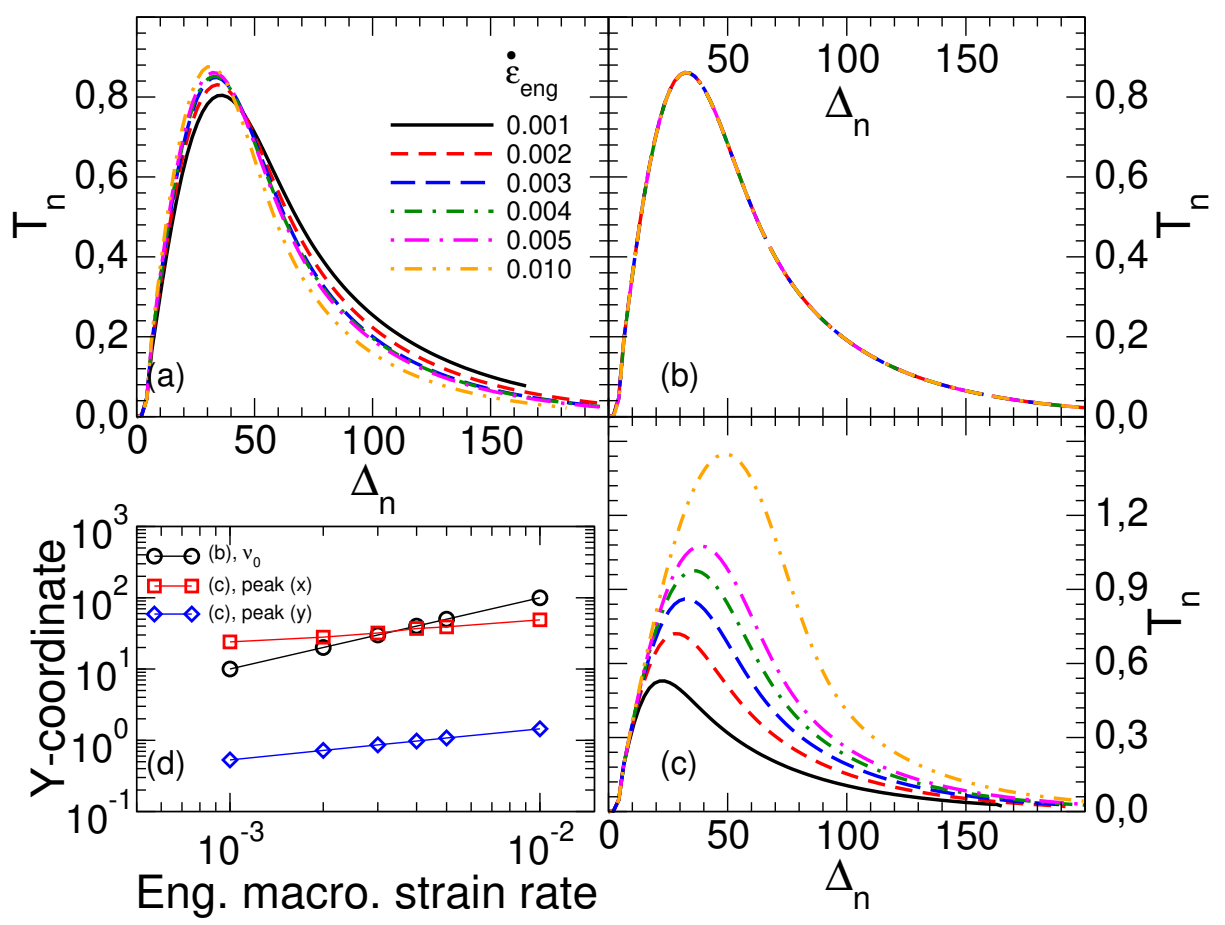

Figure 7: (Color online) There exists some well-defined shape factors to preserve at best the shape of the curves, while changing their height or their spreading. For panels (a) and (b), the engineering strain rates $\dot{\varepsilon}_{\text {eng }}$ are 0.001 , $0.002,0.003,0.004,0.005,0.010$ and, the characteristic opening $\Delta_{\mathrm{n}}^{\text {ch }}$ was fixed to $32[\sigma]$. 
To find similitude conditions like in Fluid Mechanics, let us perform a dimensional analysis on the elastoviscoplastic model. Any traction is normalized by $s_{0}$, like $s^{\star}=s / s_{0}$ for example. Any opening rate is normalized by $v_{0}$, like $v_{\mathrm{p}}^{\star}=v_{\mathrm{p}} / v_{0}$ for example. One introduces $\eta^{\star}=\eta / \eta_{\mathrm{cv}}$ as well and, a well-chosen characteristic time scale $t_{\tau}{ }^{2}$ to obtain $t^{\star}=t / t_{\tau}$. This characteristic time scale $t_{\tau}$ is not known but one can build our thinking anyway. If one introduces a well-chosen characteristic opening $\Delta_{\mathrm{n}}^{\text {ch }}$ and reminds that the (engineering) macroscopic vertical strain rate was denoted by $\dot{\varepsilon}_{\text {eng }}=\dot{\varepsilon}_{33}$, so that one has that $\Delta_{\mathrm{n}}^{\mathrm{ch}}=\dot{\varepsilon}_{\text {eng }} L_{z, 0} t_{\tau}$, one concludes that the following ratios,

$$
\begin{aligned}
& \zeta_{1}=\frac{K_{\mathrm{n}}}{s_{0}}\left(v_{0} t_{\tau}\right)=\frac{K_{\mathrm{n}} v_{0}}{s_{0}}\left(\frac{\Delta_{\mathrm{n}}^{\mathrm{ch}}}{\dot{\varepsilon}_{\text {eng }} L_{z, 0}}\right), \\
& \zeta_{2}=\frac{g_{0}}{\eta_{\mathrm{cv}}}\left(v_{0} t_{\tau}\right)=\frac{g_{0} v_{0}}{\eta_{\mathrm{cv}}}\left(\frac{\Delta_{\mathrm{n}}^{\mathrm{ch}}}{\dot{\varepsilon}_{\text {eng }} L_{z, 0}}\right), \\
& \zeta_{3}=\left(b \eta_{\mathrm{cv}}\right), \\
& \zeta_{4}=\frac{h_{0}}{s_{0}}\left(v_{0} t_{\tau}\right)=\frac{h_{0} v_{0}}{s_{0}}\left(\frac{\Delta_{\mathrm{n}}^{\mathrm{ch}}}{\dot{\varepsilon}_{\text {eng }} L_{z, 0}}\right), \\
& \zeta_{5}=s_{c v} / s_{0},
\end{aligned}
$$

should have an influence on the shape of the curve. To keep similar conditions, these ratio should stay constants in principles, so as $m_{1}$ and $m_{2}$. Since there are 10 fitting parameters and only 7 dimensionless numbers (i.e., the $\zeta_{i}, m_{1}$ and $m_{2}$ ) according to Vaschy-Buckingham's theorem, the fitting procedure would seem to be a difficult task.

To illustrate the difficulties one has to face with, let us consider the panels in Fig. 7, where one plotted the traction - opening curves for the same shape factors $\zeta_{1}=12.69, \zeta_{2}=0.186, \zeta_{3}=0.003, \zeta_{4}=0.00254$, $\zeta_{5}=6 \cdot 10^{-5}, m_{1}=0.5$ and $m_{2}=1$. In panel (a), the interface stiffness is strain-rate sensitive (i.e., it follows the arbitrary relation $\left.K_{\mathrm{n}}=0.073+\left(\log \dot{\varepsilon}_{\text {eng }}\right) / 1000\right)$, whereas it was kept fixed at $K_{\mathrm{n}}=0.05$ in panel (b). One remarks that the curves are very close (panel a) and superimposes together (panel b). It is a first nice conclusion dressed from our shape factors. A second interesting conclusion is that in panel (b), all fitting-parameters are identicals between the curves, expect for $\log \left(v_{0}\right)$, which scales like $\log \left(\dot{\varepsilon}_{\text {eng }}\right)$, see panel (d) in Fig. $7\left(v_{0}=50\right.$ for $\left.\dot{\varepsilon}_{\text {eng }}=0.005\right)$. In panel (c), still in Fig. 7, the strain-rate sensitivity is depicted for the fitting parameters taken from the curve of panel (b) at $\dot{\varepsilon}_{\text {eng }}=0.003$. In this situation, one observes an interesting behavior: the $x$-position of the maximum of the curve is proportional to $\propto\left(\dot{\varepsilon}_{\text {eng }}\right)^{0.33}$, the exponent 0.33 being so close to $m_{1} /\left[\log \left(v_{0}\right)\right]$ with $v_{0}=30$ (for $\dot{\varepsilon}_{\text {eng }}=0.003$ ); the $y$ position of the maximum of the curve is proportional to $\propto\left(\dot{\varepsilon}_{\text {eng }}\right)^{0.43}$. Let us finally consider the panels in Fig. 8, where one can observe the influence of parameters $h_{0}, b, g_{0}$ and $h_{0}$ on the shape of the curve. The tested values are given in the caption.

\section{Residue minimization and parameters optimization}

One presents here a full description of our parameters optimization. The idea hidden behind that is to find three sets of fitting parameters, since three areal densities are tested. For a fixed areal density magnitude,

\footnotetext{
${ }^{2}$ This characteristic time scale $t_{\tau}$ characterizes the speed of opening of the interface and has nothing to do, in principles, with the relaxation time $\tau_{\mathrm{r}}$ which characterizes the viscoelastic relaxation of the interface.
} 
the model should ideally fit the MD data over the complete range of opening rates. The MD data are given in Fig. 9.

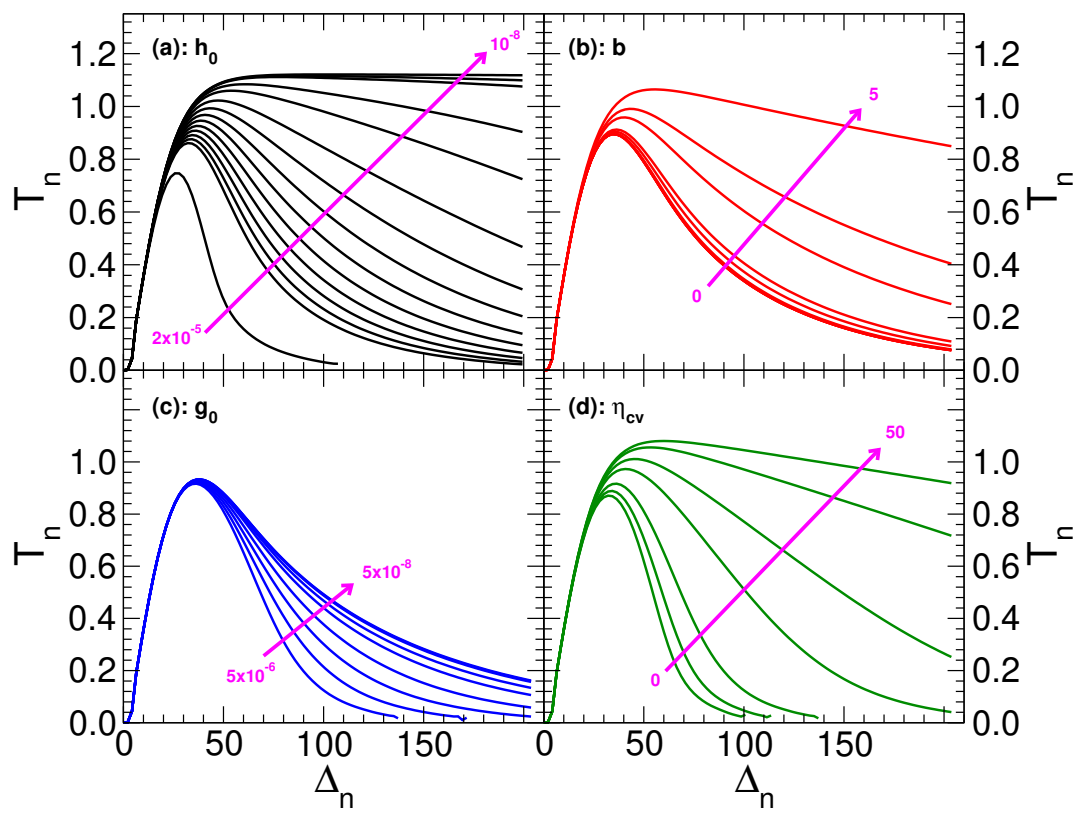

Figure 8: (Color online) Sensitivity of the proposed cohesive law to its material parameters: (a) $h_{0}$; (b) $b$; (c) $g_{0}$ and (d) $\eta_{\mathrm{cv}}$. The common parameters are: $K_{\mathrm{n}}=0.05, v_{0}=50, m_{1}=0.5, m_{2}=1.0, s_{0}=10$ and, $s_{c v}=0.0006$. The separation rate $\dot{\Delta}_{\mathrm{n}}$ is constant and equal to 0.63050 (i.e., $\dot{\varepsilon}_{\text {eng }}=0.005$ in case of pull-out separation). For panel (a) where in addition $g_{0}=1.1 \cdot 10^{-4}, b=0.002$ and $\eta_{c v}=1.5$, tested values are: $h_{0}=2 \cdot 10^{-5}, 1 \cdot 10^{-5}$, $9 \cdot 10^{-6}, 8 \cdot 10^{-6}, 7 \cdot 10^{-6}, 6 \cdot 10^{-6}, 5 \cdot 10^{-6}, 4 \cdot 10^{-6}, 3 \cdot 10^{-6}, 2 \cdot 10^{-6}, 1 \cdot 10^{-6}, 5 \cdot 10^{-7}, 1 \cdot 10^{-7}, 5 \cdot 10^{-8}, 1 \cdot 10^{-8}$ (follow the arrow). For panel (b) where in addition $g_{0}=0.0, h_{0}=8 \cdot 10^{-6}$ and $\eta_{c v}=1.5$, tested values are: $b=0.0,1 \cdot 10^{-4}$, $5 \cdot 10^{-4}, 1 \cdot 10^{-3}, 5 \cdot 10^{-3}, 1 \cdot 10^{-2}, 5 \cdot 10^{-2}, 1 \cdot 10^{-1}, 5 \cdot 10^{-1}, 1,5$ (follow the arrow). For panel (c) where in addition $b=0.25, h_{0}=8 \cdot 10^{-6}$ and $\eta_{c v}=1.5$, tested values are: $g_{0}=5 \cdot 10^{-6}, 4 \cdot 10^{-6}, 3 \cdot 10^{-6}, 2 \cdot 10^{-6}, 1 \cdot 10^{-6}, 5 \cdot 10^{-7}$, $1 \cdot 10^{-7}, 5 \cdot 10^{-8}$ (follow the arrow). For panel (d) where in addition $b=0.25, h_{0}=8 \cdot 10^{-6}$ and $g_{0}=5 \cdot 10^{-6}$, tested values are: $\eta_{c v}=0.0,0.5,1.5,5.0,10.0,25.0,50$ (follow the arrow).

\subsection{Determination of the strain-rate sensitivity}

The first important step is to find the exponant strain-rate sensitivities $m_{1}$ and $m_{2}$, since any small variation (of any of the two exponents) may induce a consequent drift during the minimization. The interface traction is maximal when $\dot{T}_{\mathrm{n}}=0$, which occurs when $\dot{\Delta}_{\mathrm{n}, \mathrm{e}}=0$, that is when,

$$
\dot{\Delta}_{\mathrm{n}}=\dot{\Delta}_{\mathrm{n}, \mathrm{p}},
$$

since one knows for sure the magnitude of $T_{\mathrm{n}}$ at the peak value. Hence according to Eq. (7), the peak traction can be written as follow,

$$
\sigma_{\mathrm{coh}}=s\left\{\sinh ^{-1}\left[\left(\frac{\dot{\Delta}_{\mathrm{n}}}{v_{0}}\right)^{m_{2}}\right]\right\}^{m_{1}},
$$


where one has also that,

$$
\sinh ^{-1}(x)=\ln \left(x+\sqrt{1+x^{2}}\right) .
$$

Eq. (30) shows how the peak traction $\sigma_{\text {coh }}$ depends on the opening rate $\dot{\Delta}_{\mathrm{n}}$. Both variables can be extracted from the MD results. For example, to be simple as a first basic picture, let us suppose that $h_{0}=0$ in Eq. (13), so that one has $s=s_{0}$ at every time. In this situation if the interface opens at $\dot{\Delta}_{\mathrm{n}}=v_{0}$, the cohesive law only reduces to a growing curve tending to a final plateau value at a height given by $\sigma_{\mathrm{coh}}=s_{0}\left[\sinh ^{-1}(1)\right]^{m_{1}}$, where $m_{1}=1$ in anticipation.

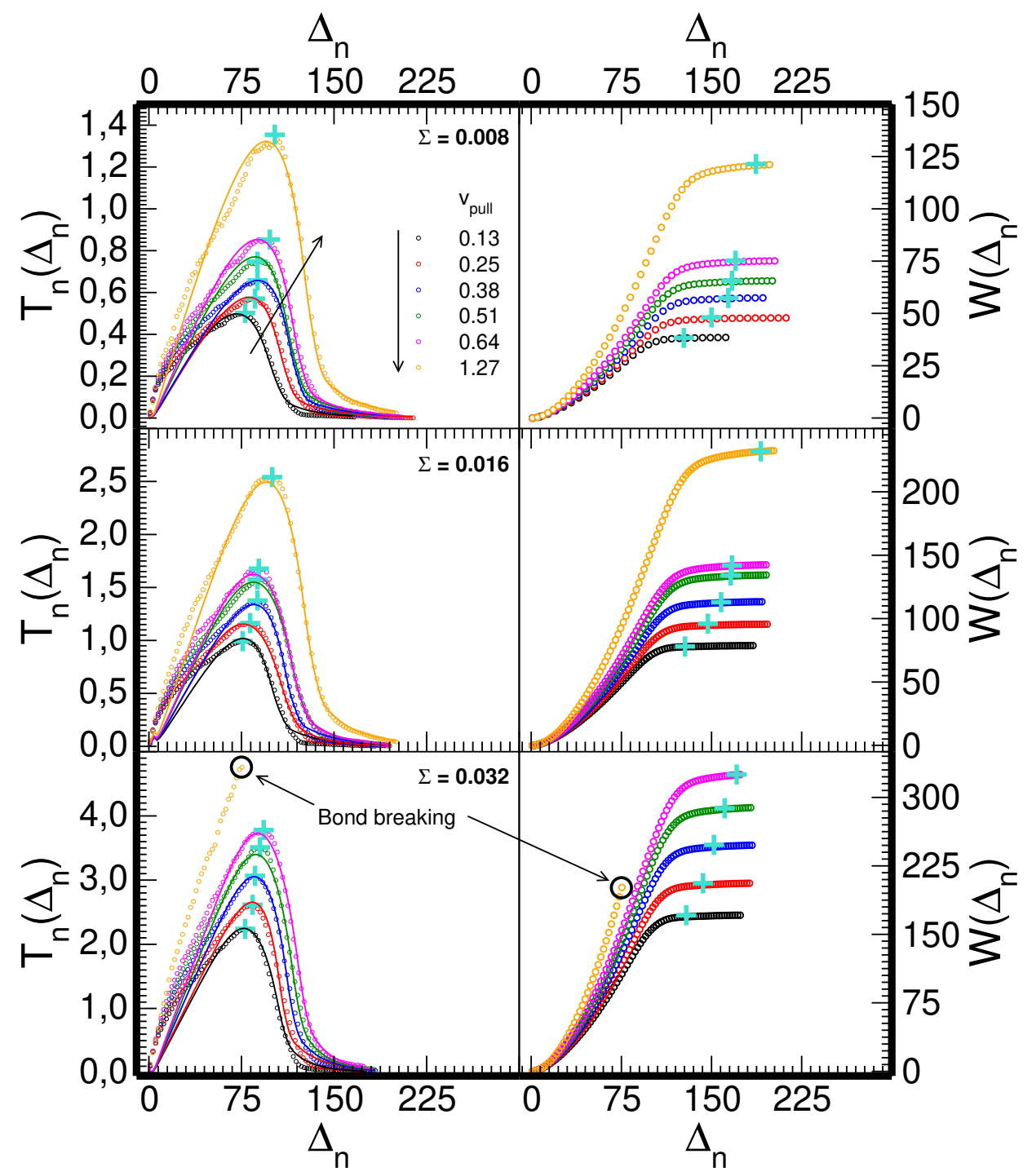

Figure 9: (Color online) MD simulations (symbols) for the three areal densities $\Sigma$ tested. The vertical traction $T_{\mathrm{n}}$, and the dissipated energy $W$ as a function of the opening $\Delta_{\mathrm{n}}$. Pure pull-out simulations are considered for reminding. A comparison of the MD results for the traction-separation response with the proposed cohesive law (lines) is proposed for different opening rates. The parameter values are given in Tab. 2. 
In order to estimate the opening-rate sensitivity exponents $m_{1}$ and $m_{2}$, let us suppose that there exists a reference set $\left(v_{0}^{\text {ref }} ; s_{0}^{\text {ref }}\right)$, choosen for an arbitray engineering macroscopic strain rate $\dot{\varepsilon}_{\text {eng }}(0.001,0.002$, $0.003,0.004,0.005$ or 0.010 ) and, let us make the following simplification (absolutely valid in pull-out),

$$
\frac{\sigma_{\mathrm{coh}}}{s_{0}^{\text {ref }}} \approx K_{0}\left\{\sinh ^{-1}\left[\left(\frac{\dot{\varepsilon}_{\text {eng }}}{\dot{\varepsilon}_{\text {eng, ref }}}\right)^{m_{2}}\right]\right\}^{m_{1}},
$$

where $\sigma_{\mathrm{coh}}=\sigma_{\mathrm{coh}}\left(\dot{\varepsilon}_{\text {eng }}\right)$ is the cohesive strength at the strain rate $\dot{\varepsilon}_{\text {eng }}$ and, where $s_{0}^{\text {ref }}$ is the cohesive strength for the reference strain rate $\dot{\varepsilon}_{\text {eng,ref }}(0.001,0.002,0.003,0.004,0.005$ or 0.010$)$. Eq. (32) is thus the fitting function to find the strain rate sensitivity. The prefactor $K_{0}$ should, as principle, be as close as possible to $\left[\sinh ^{-1}(1)\right]^{-m_{1}}$, but one lets it as being a full additional degree-of-freedom to improve the fitting procedure and, to obtain (hopefully) overall strain-rate sensitivities valid over the full range of tested opening velocities. Since one has no idea for which reference strain rate must be taken, this method was applied to each strain rate: this explains the presence of 6 panels in Fig. 10. The results are illustrated in Fig. 10, where $m_{1}$ was set to 1 by default. When the case $m_{1}=1$ is considered, one observes that the strain rate sensitivity is reasonably well captured by the Powell-Eyring law: a better accuracy was observed when $\dot{\varepsilon}_{\text {eng,ref }}$ is taken as to be 0.010 . All the results are additionally tabulated in Fig. 11, where one can notice that all values for $m_{2}$ accumulate around three averaged values depending on the areal density $\Sigma$ only.

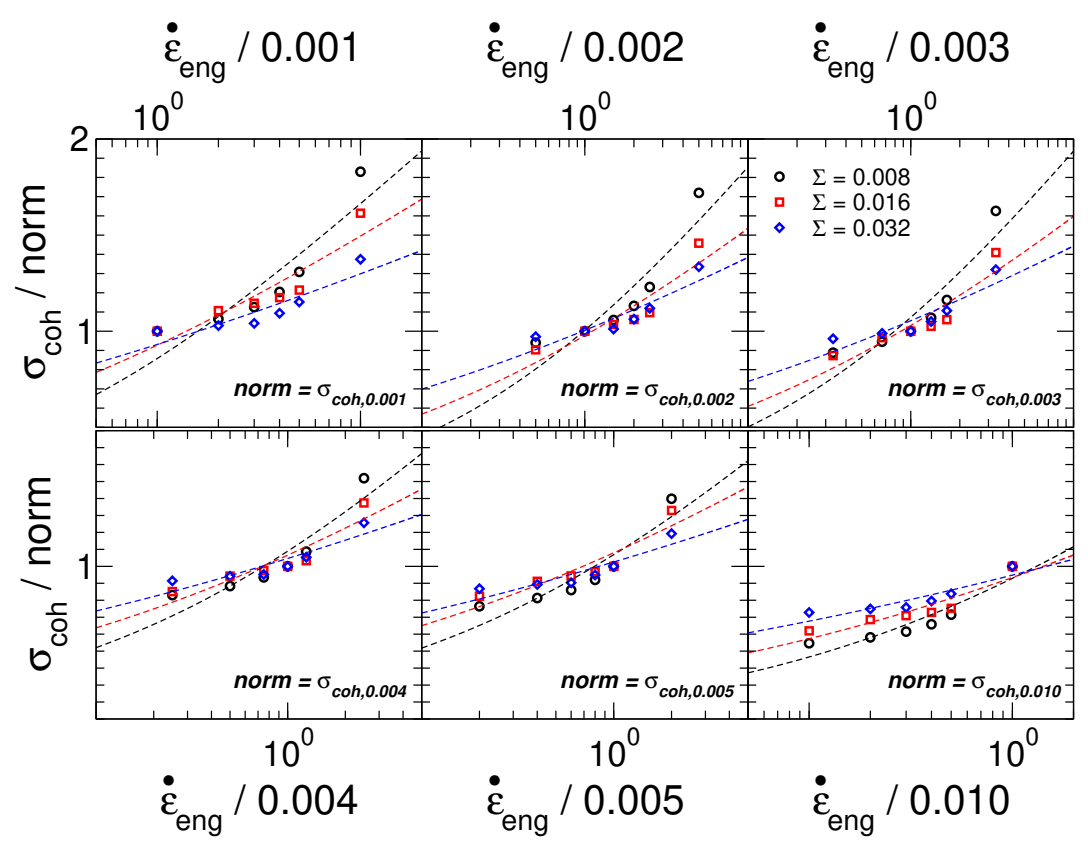

Figure 10: (Color online) The normalized cohesive strength " $\sigma_{\text {coh }} / s_{0}^{\text {ref } " ~ a s ~ a ~ f u n c t i o n ~ o f ~ t h e ~ n o r m a l i z e d ~ s t r a i n ~ r a t e ~}$ " $\dot{\varepsilon}_{\text {eng }} / \dot{\varepsilon}_{\text {eng,ref }}$ " for the three considered areal densities. As an example, the cohesive strength was normalized with respect to $s_{0}^{\text {ref }}=\sigma_{\text {coh, } 0.003}$ (i.e., the value found at $\dot{\varepsilon}_{\text {eng }}=0.003$ ), when the reference strain rate is taken as to be $\dot{\varepsilon}_{\text {eng,ref }}=0.003$. The dashed lines are regressions of the model at Eq. (30) to the data (symbols), where one sets $m_{1}=1, m_{2}$ being free to vary. All the values for $m_{2}$ are given in Fig. 11 .

The situation where $m_{1}$ and $m_{2}$ are free to vary at the same time was disregarded, since no well-defined 
minimum was found during the parameters optimization procedure (data not shown). The apparent freedom introduced by generalizing Powell-Eyring law, through the introduction of an additional exponent $m_{1}$, seems to be lost (after all) during the parameters optimization procedure. A clever-minded correlation between $m_{1}$ and $m_{2}$ must be found, but this scientific obstacle will not be discussed in the present contribution.

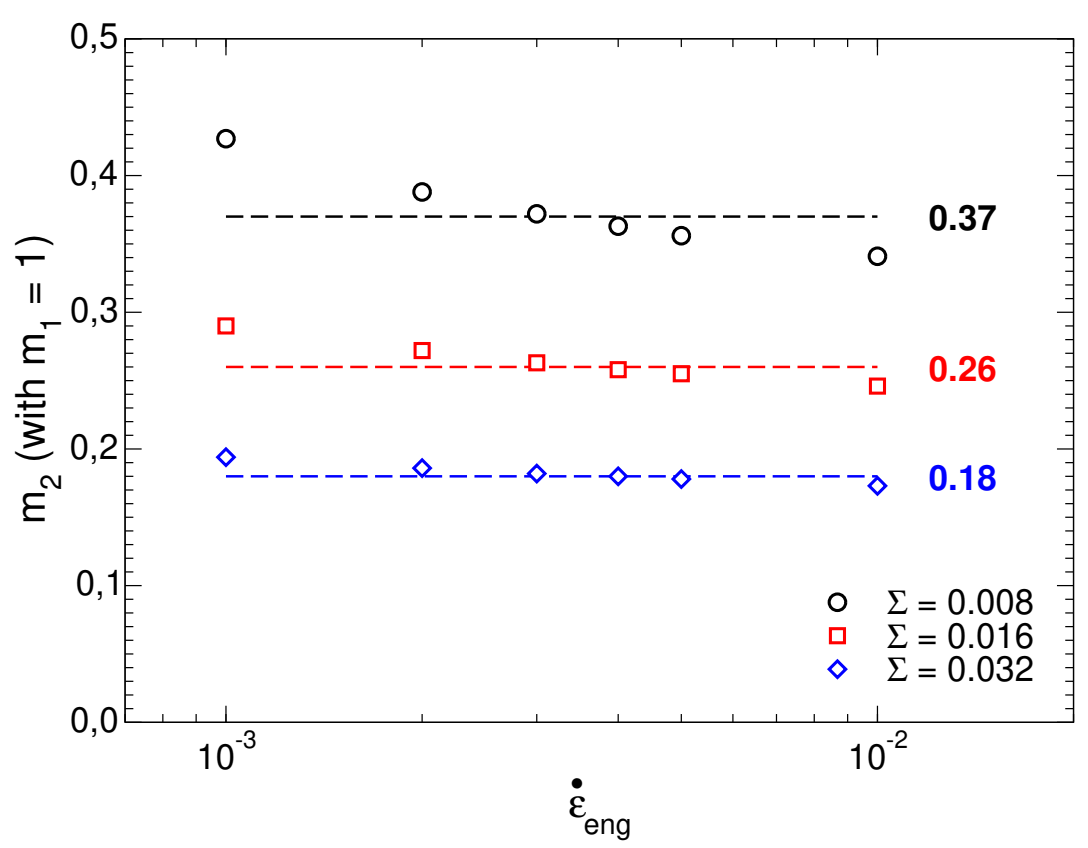

Figure 11: (Color online) The opening-rate sensitivity coefficient $m_{2}$ (for all areal densities).

\subsection{Calibrating the elasto-viscoplastic model}

One started the parameter optimization by fitting all curves separately. The confrontation between MD data and the model is given in Fig. 9 and, the material parameters being used are given in Tab. 2. The shape factors are also given in Tab. 3 as an indication for the reader. The agreement is interesting and promising: it is an evidence, since one fitted each curve one by one. According to Fig. 9, one has to adapt the interface stiffness magnitude $K_{\mathrm{n}}$ to each value of $\dot{\varepsilon}_{\text {eng }}$, however: it is constant during the cohesive opening as the basic vision of pure elasticity, but $K_{\mathrm{n}}$ is prefixed by the magnitude of the macroscopic value of $\dot{\varepsilon}_{\text {eng }}$ (closely linked to $\dot{\Delta}_{\mathrm{n}}$ in pure pull-out, see Tab. 1). Nevertheless it is gratifying to notice that for a fixed areal density, the material parameters (expect $K_{\mathrm{n}}$ ) have very similar order of magnitudes, particularly for $\dot{\varepsilon}_{\text {eng }}$ from 0.001 to 0.005 . The fastest opening rate, for which one has $\dot{\varepsilon}_{\text {eng }}=0.010$, should have definitely a different activation energy, since one observes a jump in the material constants.

\subsection{Calibrating the viscoelasto-viscoplastic model}

The elasto-viscoplastic model is interesting but, does not take into account the necessary opening-rate sensitivity for the stiffness $K_{\mathrm{n}}$. Nevertheless, the material parameters given in Tab. 2 deserves as initial 
guess for the parameter optimization, to start the minimization not so far from an optimized minimum (for which one suspected the existence). On the other hand, the viscoelasto-viscoplastic model would be able to propose a better prediction of the cohesive law during the loading. As a first simple representation of polymer interface adhesion, this model uses a single relaxation time for viscoelasticity instead of a relaxation spectrum, since the latter can include the viscoplasticity as well, if it is not adequately calibrated. To estimate the initial values of $K_{\mathrm{n}, \mathrm{u}}, K_{\mathrm{n}, \mathrm{r}}$ and $\tau_{\mathrm{r}}$, let us assume a theoretical viscoelastic loading (i.e., $T_{\mathrm{n}}$ ranging from 0 to $\sigma_{\mathrm{coh}}$ at a constant viscoelastic opening $\dot{\Delta}_{\mathrm{n}, \mathrm{e}}=\dot{\Delta}_{\mathrm{n}}=\dot{\Delta}_{0}=\mathrm{v}_{\text {pull }}$ ). In this situation, the loading can be captured by the following relation,

$$
T_{\mathrm{n}}(t)=\left[\tau_{\mathrm{r}}\left(K_{\mathrm{n}, \mathrm{u}}-K_{\mathrm{n}, \mathrm{r}}\right)\left(1-e^{-t / \tau_{\mathrm{r}}}\right)+\left(K_{\mathrm{n}, \mathrm{r}}\right) t\right] \dot{\Delta}_{0},
$$

deserving as fitting-function to estimate the $K_{\mathrm{n}, \mathrm{u}}, K_{\mathrm{n}, \mathrm{r}}$ and $\tau_{\mathrm{r}}$. During the parameter optimization then, the magnitude of $\tau_{\mathrm{r}}$ was kept as a constant (as much as possible) to avoid, hopefully, a drastic direction change in the path toward a minimum in the residue function.

\begin{tabular}{|c|c|c|c|c|c|c|c|c|}
\hline & \multicolumn{8}{|c|}{$\Sigma=0.008$ and $K_{\mathrm{n}} \approx 0.092\left(\dot{\varepsilon}_{\text {eng }}\right)^{0.355}$} \\
\hline$\dot{\varepsilon}_{\text {eng }}$ & $K_{\mathrm{n}}$ & $v_{0}$ & $s_{0}$ & $s_{c v}$ & $h_{0}$ & $g_{0}$ & $b$ & $\eta_{c v}$ \\
\hline 0.001 & 0.0101 & 1.318 & 1.650 & 0.00310 & 0.0000364 & 0.0137 & 0.00177 & 10 \\
\hline 0.002 & 0.0098 & 1.318 & 1.600 & 0.00334 & 0.0000495 & 0.0136 & 0.00135 & 10 \\
\hline 0.003 & 0.0101 & 1.318 & 1.600 & 0.00335 & 0.0000481 & 0.0136 & 0.00132 & 10 \\
\hline 0.004 & 0.0123 & 1.318 & 1.650 & 0.00331 & 0.0000417 & 0.0136 & 0.00141 & 10 \\
\hline 0.005 & 0.0135 & 1.318 & 1.650 & 0.00318 & 0.0000339 & 0.0136 & 0.00154 & 10 \\
\hline \multirow[t]{2}{*}{0.010} & 0.0190 & 1.379 & 2.042 & 0.00539 & 0.0000511 & 0.0061 & 0.00463 & 10 \\
\hline & \multicolumn{8}{|c|}{ 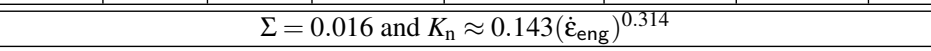 } \\
\hline$\overline{\dot{\varepsilon}_{\text {eng }}}$ & $\overline{\overline{K_{\mathrm{n}}}}$ & $\overline{v_{0}}$ & $s_{0}$ & $s_{c v}$ & $\overline{h_{0}}$ & $g_{0}$ & $\bar{b}$ & $\overline{\eta_{c v}}$ \\
\hline 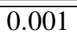 & 0.0180 & 1.285 & 2.500 & 0.00284 & 0.0000342 & 0.00300 & 0.00450 & 10 \\
\hline 0.002 & 0.0210 & 1.285 & 2.250 & 0.00285 & 0.0000219 & 0.00377 & 0.00450 & 10 \\
\hline 0.003 & 0.0210 & 1.285 & 2.500 & 0.00285 & 0.0000290 & 0.00300 & 0.00450 & 10 \\
\hline 0.004 & 0.0250 & 1.285 & 2.600 & 0.00285 & 0.0000217 & 0.00300 & 0.00450 & 10 \\
\hline 0.005 & 0.0260 & 1.285 & 2.600 & 0.00285 & 0.0000224 & 0.00300 & 0.00450 & 10 \\
\hline \multirow[t]{2}{*}{0.010} & 0.0350 & 1.285 & 3.450 & 0.00285 & 0.0000213 & 0.00300 & 0.00300 & 10 \\
\hline & \multicolumn{8}{|c|}{$\Sigma=0.032$ and $K_{\mathrm{n}} \approx 0.263\left(\dot{\varepsilon}_{\text {eng }}\right)^{0.300}$} \\
\hline$\dot{\varepsilon}_{\text {eng }}$ & $K_{\mathrm{n}}$ & $v_{0}$ & $s_{0}$ & $s_{c v}$ & $h_{0}$ & $g_{0}$ & $b$ & $\eta_{c v}$ \\
\hline 0.001 & 0.0370 & 0.903 & 3.900 & 0.00158 & 0.0000123 & 0.00113 & 0.00511 & 10 \\
\hline 0.002 & 0.0390 & 0.903 & 4.250 & 0.00170 & 0.0000167 & 0.00099 & 0.00508 & 10 \\
\hline 0.003 & 0.0445 & 0.903 & 4.500 & 0.00174 & 0.0000138 & 0.00099 & 0.00508 & 10 \\
\hline 0.004 & 0.0490 & 0.903 & 4.750 & 0.00172 & 0.0000121 & 0.00091 & 0.00507 & 10 \\
\hline 0.005 & 0.0530 & 0.903 & 5.000 & 0.00172 & 0.0000110 & 0.00085 & 0.00506 & 10 \\
\hline
\end{tabular}

Table 2: Parameter values for the curves in Fig. 9. One has also that: (a), $m_{1}=1$ and $m_{2}=0.37$ are fixed ( $\Sigma=0.008)$; (b), $m_{1}=1$ and $m_{2}=0.26$ are fixed ( $\left.\Sigma=0.016\right)$; (c), $m_{1}=1$ and $m_{2}=0.18$ are fixed $(\Sigma=0.032)$.

\begin{tabular}{|c|c|c|c|c|c|}
\hline$\Sigma$ & $\zeta_{1}$ & $\zeta_{2}$ & $\zeta_{3}$ & $\zeta_{4}$ & $\zeta_{5}$ \\
\hline \hline 0.008 & $2.4 \pm 1.4$ & $0.5 \pm 0.4$ & $0.02 \pm 0.01$ & $0.009 \pm 0.006$ & $0.0021 \pm 0.0003$ \\
\hline 0.016 & $2.8 \pm 1.7$ & $0.11 \pm 0.08$ & $0.043 \pm 0.006$ & $0.004 \pm 0.004$ & $0.0011 \pm 0.0001$ \\
\hline 0.032 & $2.6 \pm 1.5$ & $0.03 \pm 0.02$ & $0.0508 \pm 0.0002$ & $0.0008 \pm 0.0006$ & 0.0004 \\
\hline
\end{tabular}

Table 3: Shape factors for the elasto-viscoplastic cohesive law plotted from material parameters given in Tab. 2. 


\begin{tabular}{|c|c|c|c|c|c|c|c|c|c|c|}
\hline & \multicolumn{10}{|c|}{$\Sigma=0.008$} \\
\hline \hline$\dot{\varepsilon}_{\text {eng }}$ & $K_{\mathrm{n}, \mathrm{u}}$ & $K_{\mathrm{n}, \mathrm{r}}$ & $\tau_{\mathrm{r}}$ & $v_{0}$ & $s_{0}$ & $10^{3} s_{c v}$ & $10^{4} h_{0}$ & $10^{4} g_{0}$ & $10^{4} b$ & $\eta_{c v}$ \\
\hline \hline 0.001 & 0.0348 & 0.0053 & 50 & 1.454 & 2.652 & 10 & 6.36 & -1930 & 4.92 & 10.0 \\
0.002 & 0.0337 & 0.0051 & 29 & 1.354 & 2.763 & 10 & 13.0 & -456 & 108 & 10.0 \\
0.003 & 0.0285 & 0.0069 & 24 & 1.952 & 2.133 & 10 & 18.5 & +7.96 & 6090 & 9.98 \\
0.004 & 0.0283 & 0.0074 & 21 & 1.664 & 2.397 & 10 & 6.05 & -36.3 & 270 & 9.98 \\
0.005 & 0.0274 & 0.0081 & 21 & 1.640 & 2.451 & 10 & 12.2 & -7.44 & 1840 & 9.99 \\
0.010 & 0.0282 & 0.0106 & 21 & 1.736 & 2.823 & 6 & 25.2 & -0.291 & 11690 & 10.2 \\
\hline
\end{tabular}

Table 4: Parameter values for the curves in Fig. 12, where $m_{1}=1$ and $m_{2}=0.37$.

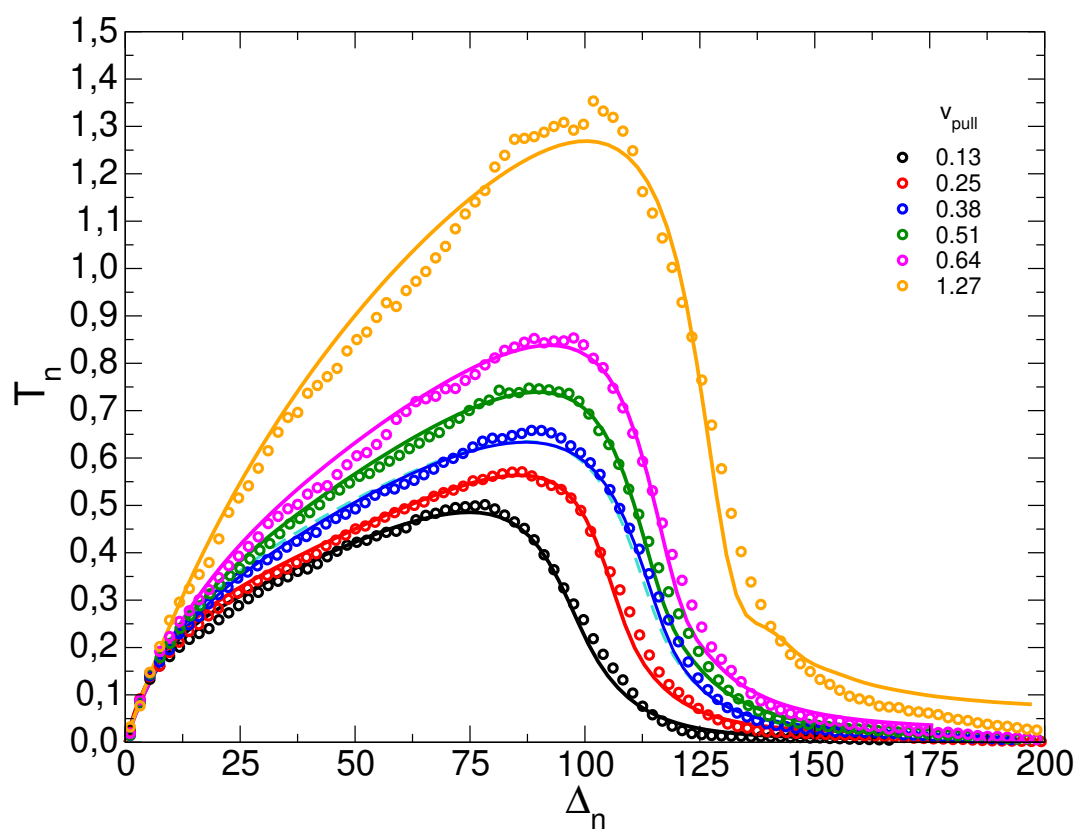

Figure 12: (Color online) MD data for cohesive laws at various engineering strain rates and, at a fixed areal density of $\Sigma=0.008$. The full visco-elasto-plastic model is plotted for the material parameters given in Tab. 4 (full lines).

\section{Discussion and Conclusion}

In Fig. 12, one provides a nice evidence that the viscoelasto-viscoplastic model is able to capture very nicely the MD data. The material parameters, used for a confrontation of the model to the MD data, are given in Tab. 4 for an areal density of $\Sigma=0.008$. One reminds that these parameters were predicted by Lagrange's principle of least action. First of all, we are glad to observe that $K_{\mathrm{n}, \mathrm{u}}$ and $K_{\mathrm{n}, \mathrm{r}}$ are very similar for all strain rates $\dot{\varepsilon}_{\text {eng }}: K_{\mathrm{n}, \mathrm{u}}=0.03 \pm 0.003$ and $K_{\mathrm{n}, \mathrm{r}}=0.01 \pm 0.002$. In addition, the relaxation time magnitudes are very similar as well, except for the lowest strain rate. Nevertheless for this lowest strain rate, the relaxation time is only doubled in comparison to the others. As a first conclusion then, our set of suitable relaxation times covers the same decade, which implies that one managed to characterize reasonably well the viscoelastic relaxation of the interface through a single time constant around 25 [LJ units]. Including a second relaxation time would be definitely a possibility to improve the characterization of the 
viscoelasticity of the interface. Furthermore, one notices that the threshold values $s_{c v}$ and $\eta_{c v}$ are almost always the same. Second of all, the parameters $v_{0}$ and $s_{0}$, governing the cohesive strength magnitude and the strain rate sensitivity (coupled with $m_{2}$ ), reach $1.6 \pm 0.2$ and $2.5 \pm 0.3$, respectively. These parameters accumulate around their own average value without any evident tendency, which would mean that these parameters would normally be a constant, but a stochastic fluctuation (correlated with any variation of other parameters) may bias the results. Last of all, the parameter optimization is more noisy for $h_{0}, g_{0}$ and $b$, which govern the shape of the decay during the unloading. In Fig. 12 and for $\dot{\varepsilon}_{\text {eng }}=0.003$, one illustrates that a second set of parameters can be found and, provides a very similar cohesive law ${ }^{3}$. This result confirms that the residue function is definitely a multi-minima function. Thus, one has to dress out a well-defined procedure to constrain the minimization into a specific region of the residue function landscape, to obtain a set of parameters maching the 6 strain rates at the same time.

In this present manuscript, we thus presented a first continuum cohesive model for polymer interface separation in the view of the simulation results we performed. One showed the possibility to find a promising agreement between the model and the simulation results. Nevertheless, one still need to improve the strategy at some points: (a), add more than one relaxation time for viscoelasticity; (b), attempt to set a spectrum of simple viscoplasticity laws, instead of playing with the exponents of a generalized Eyring's law; (c), try to fit the data from debonding simulations, see Solar [15].

\section{A A fitting procedure for none-linear regression}

Let us introduce a mathematical model, made of $N_{p}$ fitting-parameters, aimed to describe at best a set of data-points. The physical meaning of each fitting-parameter is not discussed here, but they could be choosen among the material parameters list of the cohesive law described in Sec. 4 for example. In that sense, one uses a parameter-vector representation, which represents the list of these parameters. It is denoted by: $\vec{\alpha}=\left\{\alpha_{1} ; \alpha_{2} ; \ldots ; \alpha_{N_{p}-1} ; \alpha_{N_{p}}\right\}$. According to the principle of least action in Classical Mechanics, a fitting procedure should be seen as (but not only reduces to) a minimization problem of a well-defined lagrangian $\mathcal{L}$, defined as,

$$
\mathcal{L}=-[\varepsilon(\vec{\alpha})]^{2}+\sum_{k=1}^{N_{p}} \frac{1}{2} \cdot \mathrm{m}_{k} \cdot\left(\dot{\alpha}_{k}\right)^{2},
$$

where one attributes a mass /weight $\mathrm{m}_{k}>0$ to the fitting-parameter $\alpha_{k}$. The term $\dot{\alpha}_{k}$ stands for the derivative of $\alpha_{k}$ with respect to the fitting-time-line variable $\tau$. In the most familiar procedure for none-linear regression (i.e., the so-called Levenberg-Marquardt algorithm), the second term in Eq. (34) is often neglected, but this algorithm requires matrix inversions, which very often requires CPU-time consuming operations. Instead of that, one decided to adopt a MD-like approach, where each fit-parameter is seen as a bead rolling on a curved plane, searching on an energetic well to stabilize its motion. According to Lagrange's equations of motion, one finds the equation of motion for each parameters $\alpha_{k}$ as being,

$$
\frac{\mathrm{d}}{\mathrm{d} t}\left(\frac{\partial \mathcal{L}}{\partial \dot{\alpha}_{k}}\right)-\frac{\partial \mathcal{L}}{\partial \alpha_{k}}=Q_{k}
$$

\footnotetext{
${ }^{3}$ As an example for $\dot{\varepsilon}_{\text {eng }}=0.003$, an other set of parameters (dashed line) is given and, reveals that other set of suitable parameters may be found during residue minimization: $K_{\mathrm{n}, \mathrm{u}}=0.030, K_{\mathrm{n}, \mathrm{r}}=0.0064, \tau_{\mathrm{r}}=25.0, v_{0}=1.316, m_{1}=1, m_{2}=0.37$, $s_{0}=2.00, s_{c v}=0.040, h_{0}=0.00144, g_{0}=0.0346, b=0.00502$ and $\eta_{c v}=10.0$.
} 
where $Q_{k}$ is a generalized fitting-force. One introduced a model-residue function $\varepsilon=\varepsilon(\vec{\alpha})$. For a conservative fitting-procedure, which may have an oscillating behavior however, one must set $Q_{k}=0$. For a damped fitting-procedure, which stabilizes gently into a minimum (if it exists), one must set $Q_{k}=-\zeta_{k} \cdot \dot{\alpha}_{k}$, $\zeta_{k}>0$ being a damping parameter for the fitting-parameter $\alpha_{k}$. Care must be taken with units.

\section{A.1 Theoretical presentation of the method being used}

Let us consider a single set of $N_{d}$ data-points $\left(\mathrm{x}_{j} ; \hat{\mathrm{y}}_{j}\right)$ ( $j$ ranging from 1 to $N_{d}$ ), originating here from post-processed MD simulations. This set of data-points is divided into $N_{0}$ packages of constant size $N_{1}=N_{d} / N_{0}, N_{1}$ being also an integer by assumption. For a set of given parameters, this model produces a set of $N_{d}$ model-points $\left(\mathrm{x}_{i} ; \mathrm{y}_{i}\right)$, where $\mathrm{y}_{i}=\mathrm{y}_{i}\left(\vec{\alpha} ; \mathrm{x}_{i}\right)$. Since the model must be able to describe at best the set of data-points, the most simple way to formulate the statement "to describe at best" is to write that the model is as close as possible to the set of data-points. Thus, let us introduce the model-residue function, $\varepsilon=\varepsilon(\vec{\alpha})$, defined by the following relation,

$$
\varepsilon^{2}=\sum_{i=0}^{N_{0}-1} \frac{1}{2} \cdot \chi_{i} \cdot\left[\varepsilon_{i}-\chi_{i}^{\star}\right]^{2} \quad \text { with, } \quad\left(\varepsilon_{i}\right)^{2}=\sum_{j=i \cdot N_{1}+1}^{(i+1) \cdot N_{1}}\left[\hat{y}_{j}-\mathrm{y}_{j}(\vec{\alpha})\right]^{2},
$$

where by definition " $\varepsilon>0$ " and " $\varepsilon_{i}>0$ ". The coefficients $\chi_{i}>0$ are stiffness factors and the $\chi_{i}^{\star}>0$ are subresidue equilibrium levels, aimed to force more or less the parameter-fitting procedure on some specific regions of the set of data-points. Our residue function must then be as close as possible to zero and, the fitting procedure must reach a stationary point with respect to the vector $\vec{\alpha}$. If one now calculates Eq. (35), one obtains the equation of the dynamics for each $\alpha_{k}$, that is,

$$
\begin{aligned}
\mathrm{m}_{k} \cdot \ddot{\alpha}_{k} & =\mathrm{f}_{k}-\zeta_{k} \cdot \dot{\alpha}_{k} \quad \text { where, } \quad \mathrm{f}_{k}=-\sum_{i=0}^{N_{0}-1} \chi_{i} \cdot\left[\varepsilon_{i}-\chi_{i}^{\star}\right] \cdot \frac{\partial \varepsilon_{i}}{\partial \alpha_{k}}=\frac{\partial \mathcal{L}}{\partial \alpha_{k}}, \\
\frac{\partial \varepsilon_{i}}{\partial \alpha_{k}} & =\lim _{\Delta \alpha_{k} \rightarrow 0}\left(\frac{\left[\varepsilon_{i}\left(\ldots ; \alpha_{k}+\Delta \alpha_{k} ; \ldots\right)-\varepsilon_{i}\left(\ldots ; \alpha_{k}-\Delta \alpha_{k} ; \ldots\right)\right]}{2 \cdot \Delta \alpha_{k}}\right),
\end{aligned}
$$

where $k$ runs from 1 to $N_{p}$ and, $\Delta \alpha_{k}$ is a small finite increment for the $\alpha_{k}$ fitting parameter.

Remark 1 It might be a possibility to further use the method for a residue function computed not only from the MD-data for one strain rate, but from the MD-data for all strain rates at the same time. The model-residue function $\varepsilon=\varepsilon(\vec{\alpha})$, see Eq. (36), must be then modified in accordance. Let us then consider a set of $N_{d, i}$ data-points $\left(\mathrm{x}_{k, i}, \hat{\mathrm{y}}_{k, i}\right)$ ( $k$ ranging from 1 to $\left.N_{d, i}\right)$, originating here from the post-processed $M D$ simulation of index “ $i$ " (i.e., curve $i$ ). This set of data-points is divided into $N_{0, i}$ packages of constant size $N_{1, i}=N_{d, i} / N_{0, i}, N_{1, i}$ being also an integer by assumption. Let us introduce a mathematical model, still made of $N_{p}$ fitting-parameters, aimed to describe at best this set of data-points. For a set of given parameters, this model produces a set of $N_{d, i}$ model-points $\left(\mathrm{x}_{k, i} ; \mathrm{y}_{k, i}\right)$. If $N_{c}$ stands now for the number of curves to fit (i.e., before one has set $N_{c}=1$ and $i=1$ ), the model-residue function may be re-written as,

$$
\begin{aligned}
\varepsilon^{2}=\sum_{i=1}^{N_{c}} \frac{1}{2} \cdot \bar{\chi}_{i} \cdot\left[\bar{\varepsilon}_{i}-\chi_{i}^{\star \star}\right]^{2} \quad \text { with, } \quad\left(\bar{\varepsilon}_{i}\right)^{2}=\sum_{j=0}^{N_{0, i}-1} \frac{1}{2} \cdot \chi_{j} \cdot\left[\varepsilon_{j}-\chi_{j}^{\star}\right]^{2} \\
\text { and, } \quad\left(\varepsilon_{j}\right)^{2}=\sum_{k=j \cdot N_{1, i}+1}^{(j+1) \cdot N_{1, i}}\left[\hat{y}_{k, i}-\mathrm{y}_{k, i}(\vec{\alpha})\right]^{2},
\end{aligned}
$$


where still by definition " $\varepsilon>0$ " and " $\bar{\varepsilon}_{i}>0$ ". The coefficients $\bar{\chi}_{i}>0$ are stiffness factors and the $\chi_{i}^{\star \star}>0$ are sub-residue equilibrium levels, aimed to force more or less the parameter-fitting procedure on some specific curves of the set of curves. The rest of the method remains identical, expect that the force $f_{k}$ on each parameter, see Eq. (35), must be modified in accordance and may be re-written as,

$$
\mathrm{f}_{k}=-\sum_{i=1}^{N_{c}} \sum_{j=0}^{N_{0, i}-1} \frac{1}{2} \cdot \bar{\chi}_{i} \cdot \chi_{j} \cdot \frac{\left[\bar{\varepsilon}_{i}-\chi_{i}^{\star \star}\right]}{\bar{\varepsilon}_{i}} \cdot\left[\varepsilon_{j}-\chi_{j}^{\star}\right] \cdot \frac{\partial \varepsilon_{j}}{\partial \alpha_{k}} .
$$

\section{A.2 Description of a computational strategy}

The Beeman's algorithm was used to integrate the equations of motion for each fitting-parameters $\alpha_{k}$. It is given by the literature as,

$$
\begin{aligned}
& \vec{\alpha}(\tau+\delta \tau)=\vec{\alpha}(\tau)+\dot{\vec{\alpha}}(\tau) \cdot \delta \tau+\left[\frac{2}{3} \cdot \ddot{\vec{\alpha}}(\tau)-\frac{1}{6} \cdot \ddot{\vec{\alpha}}(\tau-\delta \tau)\right] \cdot(\delta \tau)^{2}, \\
& \dot{\vec{\alpha}}(\tau+\delta \tau)=\dot{\vec{\alpha}}(\tau)+\left[\frac{1}{3} \cdot \ddot{\vec{\alpha}}(\tau+\delta \tau)+\frac{5}{6} \cdot \ddot{\vec{\alpha}}(\tau)-\frac{1}{6} \cdot \ddot{\vec{\alpha}}(\tau-\delta \tau)\right] \cdot \delta \tau .
\end{aligned}
$$

If one defines an integration time-step $\delta \tau$ for the fitting procedure, the standard procedure used to perform the fitting procedure reduces as follow. The fitting-time-line variable $\tau$ has nothing to do with the (real) time during the MD simulation producing the set of data-points $\left(x_{i} ; \hat{y}_{i}\right)$. It deals rather with the duration over which the set of parameters flows from an out-of-equilibrium position to a new position, where the protagonist estimates that this set of parameters reached a well-appreciated position of equilibrium (i.e., the model came close to the data, in the frame of the CPU time effort one allocated to the fitting procedure), see below.

Require: the vectors $\vec{\alpha}(\tau), \dot{\vec{\alpha}}(\tau), \ddot{\vec{\alpha}}(\tau-\delta \tau)$

Compute: cohesive law for $\vec{\alpha}(\tau), \varepsilon_{i}$ and residue $\varepsilon^{2}$ using Eq. (36)

Compute: drag force " $\zeta_{k} \cdot \dot{\alpha}_{k}$ " and " $\mathrm{f}_{k}$ ", see Eq. (37), for each $\alpha_{k}$ at $\tau$

Compute: vector $\ddot{\vec{\alpha}}(\tau)$ using Eq. (37)

Compute: vector $\vec{\alpha}(\tau+\delta \tau)$ using Eq. (42)

Compute: pre-estimation $\dot{\vec{\alpha}}(\tau+\delta \tau) \approx \dot{\vec{\alpha}}(\tau)+0.5 \cdot \ddot{\vec{\alpha}}(\tau) \cdot \delta \tau$

Compute: drag force " $\zeta_{k} \cdot \dot{\alpha}_{k}$ " and " $\mathrm{f}_{k}$ ", see Eq. (37), for each $\alpha_{k}$ at $\tau+\delta \tau$

Compute: vector $\ddot{\vec{\alpha}}(\tau+\delta \tau)$ using Eq. (37)

Compute: vector $\dot{\vec{\alpha}}(\tau+\delta \tau)$ using Eq. (43)

Require: update $\tau+\delta \tau \rightarrow \tau$

Table 5: Updating procedure for our fitting-procedure $\left(\delta \alpha_{k}=\dot{\alpha}_{k} \cdot \delta \tau\right)$. To start the procedure, one sets initial guesses for vectors $\vec{\alpha}(\tau=0)$ and $\dot{\vec{\alpha}}(\tau=0)$ and, sets the parameters $N_{0}, \chi_{i}$ and $\chi_{i}^{\star}\left(i\right.$ from 1 to $\left.N_{0}\right)$ and, the parameters $\mathrm{m}_{k}$ and $\zeta_{k}\left(k\right.$ from 1 to $\left.N_{p}\right)$.

\section{A.3 One example of the minimization process}

To illustrate briefly our method, one provides a minimization experiment for MD data at an engineering strain rate of 0.001 and for $\Sigma=0.008$. The idea is to illustrate the beam of curves obtained, as the 
Lagrangian equation are integrated. Thus let us define the vector of parameters as being

$$
\vec{\alpha}=\left\{K_{\mathrm{n}, \mathrm{u}} ; K_{\mathrm{n}, \mathrm{r}} ; \tau_{\mathrm{r}} ; v_{0} ; m_{1} ; m_{2} ; s_{0} ; s_{c v} ; h_{0} ; g_{0} ; b ; \eta_{c v}\right\} .
$$

Their mass $\mathrm{m}_{k}$ are all fixed to 1.0 , expect for $\tau_{\mathrm{r}}, m_{1}, m_{2}$ and $s_{c v}$, for which $\mathrm{m}_{k}=10^{7}$. The friction coefficients were all fixed to $\zeta_{k}=0.1$. The curve was sub-divided into $N_{0}=5$ packages, with: $\chi_{1}=100$, $\chi_{1}^{\star}=0.001, \chi_{j}=1$ and $\chi_{j}^{\star}=0.001(j=2,3,4$ or 5$)$. The minimization was runned over $10^{5}$ increments, with a timestep of $10^{-6}$. The intermediate results are written every $10^{4}$ increments, see Fig. 13.

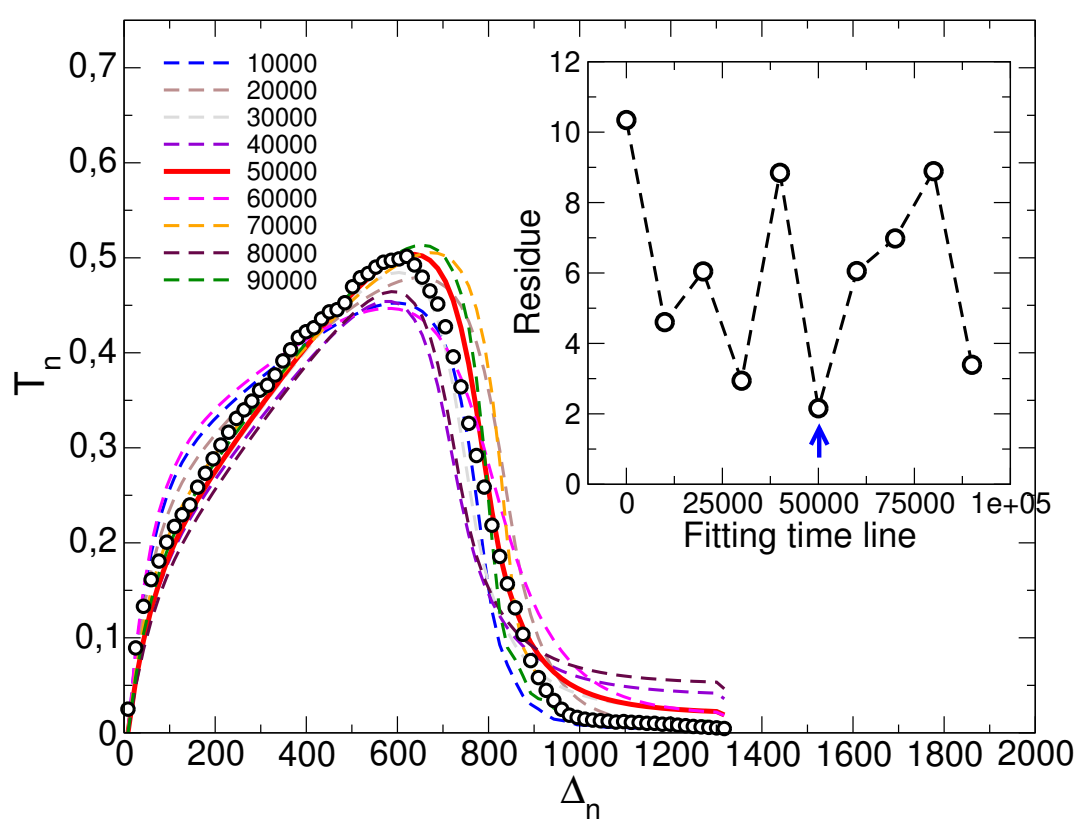

Figure 13: (Color online) The beam of curves obtained during the minimization, to adjust our cohesive law to a set of MD data. The small panel indicates the residue, as the minimization sets in: curve 6 seems to be the one, which presents the most interesting agreement.

\section{REFERENCES}

[1] C. Creton, E.J. Kramer, C.-Y. Hui and H.R. Brown, Macromolecules 25 (1992) 3075.

[2] C.-Y. Hui, A. Ruina, C. Creton and E.J. Kramer, Macromolecules 25 (1992) 3948.

[3] J. Wanhiymnn, E.J. Kramer and C.-Y. Hui, Macromolecules 26 (1993) 2928.

[4] J. Wanhiymnn, C. Creton, E.J. Kramer, F. Xiao and C.-Y. Hui, Macromolecules 26 (1993) 6011.

[5] J. Wanhiymnn, E.J. Kramer, C. Creton and C.-Y. Hui, Macromolecules 27 (1994) 2019.

[6] C. Creton, E.J. Kramer, H.R. Brown and C.-Y. Hui, Advances in Polymer Science 156 (2001) 53.

[7] A. Needleman, Applied Mechanics Review 43 (1990) 274.

[8] A. Karma, D.A. Kessler, H. Levine, PRL 87 (2001) 045501.

[9] A.W. Adamson, Physical Chemistry of Surfaces, John Wiley \& Sons Inc., USA (1997). 
[10] R.A. Veselovsky, V.N. Kestelman, Adhesion of Polymers, McGraw-Hil, USA (2002).

[11] C. Nguyen, A.J. Levy, International Journal of Solids and Structures 46 (2009) 2712.

[12] M. Bulacu and E. Van der Giessen, Journal of Chemical Physics 131 (2009) 064904.

[13] M. Bulacu and E. Van der Giessen, European Physics Letters 93 (2011) 63001.

[14] M. Solar and E. van der Giessen, Computational Materials Science (2012).

[15] M. Solar, Computational Materials Science 186 (2021) 110048.

[16] M. Parrinello and A. Rahman, Journal of Applied Physics 52 (1981) 7182.

[17] Schnell B., Ph.D. thesis in Polymer Physics. Strasbourg (France): thesis UdS, 2006, http://eprintsscd-ulp.u-strasbg.fr:8080/545/.

[18] Allen M. P., Tildesley D. J., Computer Simulation of Liquids, Oxford Science Publications, USA (1987).

[19] Lemaitre J., A Course on Damage Materials, Springer-Verlag, Berlin Heidelberg (1996).

[20] M. Bulacu and E. Van der Giessen, Physical Review E 76 (2007) 011807.

[21] M. Bulacu and E. Van der Giessen, The Journal of Chemical Physics 123 (2005) 114901.

[22] R. Auhl, R. Everaersa, G.S. Grest, K. Kremer and S.L. Plimpton, Journal of Chemical Physics 119 (2003) 12718.

[23] G.S. Grest and K. Kremer, Physical Review A 33 (1986) 3628.

[24] E. Raphael and P.G. de Gennes, Journal of Physical Chemistry 96 (1992) 4002.

[25] Frenkel D., Smit B., Understanding Molecular Simulation from Algorithms to Applications, Academic Press, UK (1996).

[26] M. Dressler and B.J. Edwards, International Journal of Modern Physics C 13 (2002) 1273.

[27] H.J.M. Hanley and D.J. Evans, International Journal of Thermophysics 11 (1990) 381.

[28] B.J. Edwards and M. Dressler, Journal of Non-Newtonian Fluid Mechanics 96 (2001) 163.

[29] W.G. Hoover, Annual Review of Physical Chemistry 34 (1983) 103.

[30] W.G. Hoover and C.G. Hoover, Condensed Matter Physics 8 (2005) 247.

[31] W.G. Hoover, D.J. Evans, R.B. Hickman, A.J.C. Ladd and B. Moran, Physical Review A 22 (1980) 1690.

[32] T. Soddemann, B. Dünweg and K. Kremer, Physical Review E 68 (2003) 046702.

[33] B. Liu and X. Qiu, Journal of Computational and Theoretical Nanoscience 6 (2009) 1081.

[34] D.K. Mahajan and S. Basu, International Journal of Applied Mechanics 2 (2010) 515.

[35] F. Costanzo, G.L. Gray and P.C. Andia, Modeling and Simulation in Materials Science and Engineering 12 (2004) S333.

[36] J.H. Rose, J.R. Smith, J. Ferrante, Physical Review B 28 (1983) 4.

[37] J.H. Rose, J. Ferrante, J.R. Smith, Physical Review Letters 47 (1981) 9. 
[38] R.L. Hayes, M. Ortiz, E.A. Carter, Physical Review B 69 (2004) 172104.

[39] A. Banerjea, J.R. Smith, Physical Review B 37 (1988) 37.

[40] O. Nguyen, M. Ortiz, Journal of the Mechanics and Physics of Solids 50 (2002) 1727.

[41] L. Anand, M.E. Gurtin, International Journal of Solids and Structures 40 (2003) 1465-1487.

[42] Ward I M, Sweeney J: An introduction to the mechanical properties of solid polymers. John Wiley and Sons, Ltd UK (2004).

[43] McCrum N G, Buckley C P, Bucknall C B: Principles of polymer engineering. Oxford University Press, New York (1988).

[44] Treloar L R G: The physics of rubber elasticity. Clarendon Press Oxford, UK (1975).

[45] G.F. Wang, E. van der Giessen, European Journal of Mechanics and Solids 23 (2004) 395.

[46] S. Basu, D.K. Mahajana, E. van der Giessen, Polymer 46 (2005) 7504.

[47] Th. Seelig, E. van der Giessen, Computational Materials Science 45 (2009) 725.

[48] Gradshteyn I.S., Ryzhik I.M., Table of Integrals, Series, and Products, Academic Press, USA (2007). 